\title{
Top-Yukawa contributions to bbH production at the LHC
}

\author{
Nicolas Deutschmann, ${ }^{a}$ Fabio Maltoni, ${ }^{b}$ Marius Wiesemann ${ }^{c}$ and Marco Zaro ${ }^{d}$ \\ ${ }^{a}$ Institute for Theoretical Physics, ETH Zurich, \\ 8093 Zurich, Switzerland \\ ${ }^{b}$ Centre for Cosmology, Particle Physics and Phenomenology (CP3), \\ Université catholique de Louvain, B-1348 Louvain-la-Neuve, Belgium \\ ${ }^{c}$ Theoretical Physics Department, CERN, \\ Geneva, Switzerland \\ ${ }^{d}$ Nikhef, Science Park 105, NL-1098 XG Amsterdam, The Netherlands \\ E-mail: ndeutschmann@itp.phys.ethz.ch, fabio.maltoni@uclouvain.be, \\ marius.wiesemann@cern.ch, m.zaro@nikhef.nl
}

ABSTRACT: We study the production of a Higgs boson in association with bottom quarks $(b \bar{b} H)$ in hadronic collisions at the LHC, including the different contributions stemming from terms proportional to the top-quark Yukawa coupling $\left(y_{t}^{2}\right)$, to the bottom-quark one $\left(y_{b}^{2}\right)$, and to their interference $\left(y_{b} y_{t}\right)$. Our results are accurate to next-to-leading order in QCD, employ the four-flavour scheme and the (Born-improved) heavy-top quark approximation. We find that next-to-leading order corrections to the $y_{t}^{2}$ component are sizable, making it the dominant production mechanism for associated $b \bar{b} H$ production in the Standard Model and increasing its inclusive rate by almost a factor of two. By studying final-state distributions of the various contributions, we identify observables and selection cuts that can be used to select the various components and to improve the experimental sensitivity of $b \bar{b} H$ production on the bottom-quark Yukawa coupling.

KEYwORDS: NLO Computations

ARXiv EPRINT: 1808.01660 


\section{Contents}

1 Introduction 1

2 Outline of the calculation 4

2.1 Coupling structure of the $b \bar{b} H$ cross section 4

2.2 HEFT approximation in $b \bar{b} H$ production 6

2.3 Assessment of the HEFT approximation 8

3 Phenomenological results 10

$\begin{array}{lll}3.1 & \text { Input parameters } & 10\end{array}$

3.2 Predictions for $b \bar{b} H$ production in the SM 11

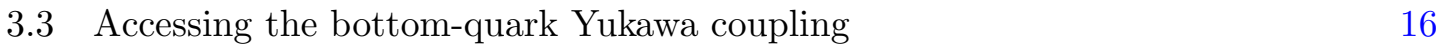

$\begin{array}{lll}3.4 & \text { QCD corrections to } g \rightarrow b \bar{b} \text { splitting } & 18\end{array}$

4 Summary and outlook $\quad 22$

A Jet rates with $R=1 \quad 24$

B Matching and renormalization schemes $\quad 25$

C The HEFT bottom-quark Yukawa at $\mathcal{O}\left(1 / m_{t}\right) \quad 28$

$\begin{array}{ll}\text { D Master integrals } & 31\end{array}$

\section{Introduction}

After the discovery of a scalar resonance with a mass of about $125 \mathrm{GeV}[1,2]$, the accurate determination of its couplings to Standard-Model (SM) particles has become one of the major objectives of LHC Run II and beyond. Data collected at the LHC so far supports the hypothesis that this resonance is the scalar boson predicted by the Brout-EnglertHiggs mechanism of electroweak symmetry breaking $[3,4]$ as implemented in the SM [5]: the Higgs couplings are universally set by the masses of the corresponding particles the Higgs boson interacts with. Global fits of various production and decay modes of the Higgs boson [6-10] constrain its couplings to third-generation fermions and to vector bosons to be within $10-20 \%$ of the values predicted by the SM. In particular, the recent measurement of Higgs production in association with a top-quark pair [11, 12] provides the first direct evidence of the coupling between the Higgs boson and the top quark, thereby proving that gluon-gluon Higgs production proceeds predominantly via top-quark loops. The coupling of the Higgs boson to $\tau$ leptons has also been established at the $5 \sigma$ level for some time $[13,14]$, while the Higgs coupling to bottom quarks has been observed only very recently [15]. By contrast, to date, we have no experimental confirmation that the Higgs boson couples to first-/second-generation fermions, nor about the strength of the Higgs self-interaction. 
The ability to probe elementary couplings and to improve the experimental sensitivity strongly relies on precise theoretical predictions for both production and decay. The bottom-quark Yukawa coupling $\left(y_{b}\right)$ plays a rather special role in this context: despite having a relatively low coupling strength with respect to the couplings to vector bosons and top quarks, the $H \rightarrow b \bar{b}$ decay dominates the total decay width in the SM for a Higgs-boson mass of about $125 \mathrm{GeV}$ due to kinematical and phase space effects. The observation of this decay is, however, quite challenging because of large backgrounds generated by QCD, especially in the gluon-fusion production mode [8], and has for now only been searched for in vector-boson fusion $[16,17]$ and Higgsstrahlung $[8,18]$. The latter is the most sensitive channel, yielding a signal strength for the decay branching ratio of $\mu_{b b}=1.0 \pm 0.2$ [15]. However, since the total Higgs width is dominated by $H \rightarrow b \bar{b}$, the corresponding branching ratio has a rather weak dependence on $y_{b}$. As a result, the sensitivity of processes involving Higgs decays to bottom quarks on this parameter is in fact rather low.

Studying production modes featuring a $b \bar{b} H$ coupling is a promising alternative: on the one hand, Higgs production in the SM (inclusive over any particles produced in association) proceeds predominantly via the gluon-fusion process, where the Higgs-gluon coupling is mediated by heavy-quark loops. In particular, bottom-quark loops have a contribution of about $-6 \%$ to the inclusive cross section, which can become as large as $-10 \%$ for Higgs bosons produced at small transverse momentum [19-26]. On the other hand, the associated production of a Higgs boson with bottom quarks ( $b \bar{b} H$ production) provides direct access to the bottom-quark Yukawa coupling already at tree-level [27]. It yields a cross section comparable to the one of the associated production with top quarks (roughly $0.5 \mathrm{pb}$ at $13 \mathrm{TeV}$ ), which is about $1 \%$ of the fully-inclusive Higgs-production rate in the SM. Furthermore, the inclusive rate decreases dramatically once conditions on the associated $b$ jets are imposed to make it distinguishable from inclusive Higgs-boson production.

The SM picture outlined above might be significantly modified by beyond-SM effects: while a direct observation in the SM is challenging at the LHC, $b \bar{b} H$ production plays a crucial role in models with modified Higgs sectors. In particular in a generic two Higgsdoublet-model (2HDM), or in a supersymmetric one such as the MSSM, the bottom-quark Yukawa coupling can be significantly increased, promoting $b \bar{b} \phi$ to the dominant Higgs production mode $[28,29]$ in many benchmark scenarios, $\phi$ being any of the scalars or pseudo-scalars in such theories. Given that a scalar sector richer than that of the SM has not yet been ruled out experimentally, this is a fact that one must bear in mind, and that constitutes a strong motivation for theoretical studies of scalar-particle production in association with bottom quarks.

The production of $b \bar{b} H$ final states receives additional contributions from the loopinduced gluon-fusion process (proportional to $y_{t}^{2} ; y_{t}$ being the top-quark Yukawa coupling), which in the SM is of similar size as $y_{b}^{2}$ contributions, but have rarely been studied in the literature. In this paper, we consider Higgs production in association with bottom quarks for all contributions proportional to $y_{b}^{2}$ and $y_{t}^{2}$ at NLO QCD, as well as their interference terms proportional to $y_{b} y_{t}$. The $b \bar{b} H$ process is particularly interesting also from a theoretical viewpoint in many respects. First, as for all mechanisms that feature bottom quarks at the level of the hard process, there are two schemes applicable to performing 
the computation. These so-called four-flavour scheme (4FS) and five-flavour scheme (5FS) reflect the issue that arise from different kinematic regimes, where either the mass of the bottom-quark can be considered a hard scale or bottom quarks are treated on the same footing as the other light quarks. Hence, the bottom-quark is considered to be massive in the $4 \mathrm{FS}$, while its mass can be set to zero in the $5 \mathrm{FS}$. The advantages of either scheme in the context of $b \bar{b} H$ production have been discussed in detail in ref. [30]. We employ the $4 \mathrm{FS}$ throughout this paper, owing to its superior description of differential observables related to final-state bottom quarks and the definition of bottom-flavoured jets, which is particularly striking in fixed-order computations. Another theoretical motivation lies in the nature of the loop-induced gluon-fusion process that leads to the contributions proportional to $y_{t}^{2}$. Being dominated by kinematical configurations where the Higgs boson recoils against a gluon which splits into a bottom-quark pair, this collider process features the cleanest and most direct access to $g \rightarrow b \bar{b}$ splittings. Thus, as a bonus, our computation also allows us to study the effect of NLO corrections on such splittings.

Given that the NLO QCD corrections to $b \bar{b} H$ production for $y_{b}^{2}$ contributions (and the LO $y_{b} y_{t}$ terms) were studied in great detail in ref. [30], including the effect of parton showers, we focus here on the computation of NLO QCD corrections to the terms proportional to $y_{t}$ and analyse their behaviour with respect to the $y_{b}^{2}$ contribution. We note that our computation of NLO corrections to the $y_{b} y_{t}$ and $y_{t}^{2}$ terms employs an effective field theory, where the top quark is integrated out from the theory and the Higgs directly couples to gluons, to which we refer as Higgs Effective Field Theory (HEFT). Besides a detailed description of the application of this approach to our problem, we will show that this approximation is quite accurate in the bulk of the phase space region which is relevant for this study.

Before introducing our calculation in the next section, we briefly summarise the status of the results for $b \bar{b} H$ production available in the literature. As far as $4 \mathrm{FS}$ computations are concerned, seminal NLO QCD fixed-order parton-level predictions were obtained in refs. [29, 31], and later updated to the case of MSSM-type couplings [32], and to SUSYQCD corrections in the MSSM [33, 34]. Part of the NLO electroweak corrections were also obtained recently in ref. [35]. The presentation of differential results in these papers is very limited as the focus is on the total cross section. Given that computations in the 5FS are technically much simpler, far more results in this scheme exist in the literature: the total cross section are known at NLO [36, 37] since a long time and even NNLO QCD [38] predictions were among the first computations at this level of accuracy ever achieved relevant for LHC phenomenology. Parton-level distributions were obtained at NLO for $H+b$ and $H+$ jet production [39, 40], and at NNLO for jet rates [41] and fully differential distributions [42]. The analytical transverse-momentum spectrum of the Higgs boson was studied up to $\mathcal{O}\left(\alpha_{s}^{2}\right)$ in ref. [43], while analytically resummed NLO+NLL and NNLO+NNLL results were presented in ref. [44] and ref. [45], respectively. ${ }^{1}$ NLO+PS predictions for both the $4 \mathrm{FS}$ and the 5FS were presented for the first time in ref. [30], including a comprehensive comparison of the two schemes and the discussion several differential distributions with NLO QCD accuracy. Other NLO+PS results were later obtained in Powheg [48] and

\footnotetext{
${ }^{1}$ Even the ingredients for the full $\mathrm{N}^{3} \mathrm{LO}$ prediction are already available [46, 47]; their combination is far from trivial though.
} 


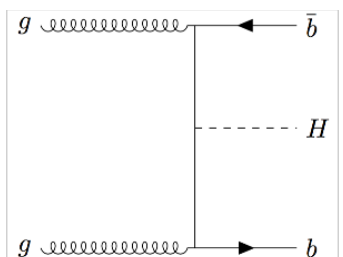

(a) $\mathcal{A}_{b}^{(0)}$

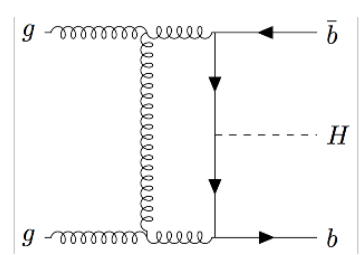

(b) $\mathcal{A}_{b}^{(1 V)}$

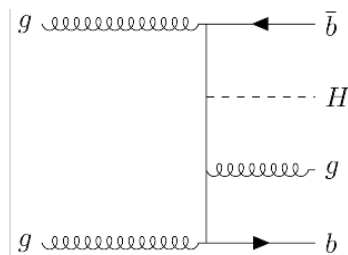

(c) $\mathcal{A}_{b}^{(1 R)}$

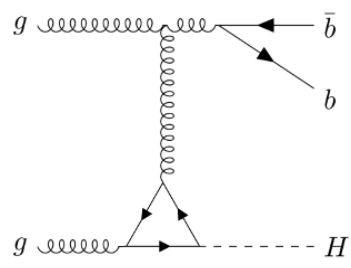

(d) $\mathcal{A}_{t}^{(0)}$

Figure 1. Examples of Feynman diagrams for $b \bar{b} H$ production at LO and at NLO, which contain virtual and real diagrams proportional to $y_{b}$, and virtual diagrams with a top loop proportional to $y_{t}$. The corresponding amplitudes are named $\mathcal{A}_{b}^{(0)}, \mathcal{A}_{b}^{(1 V)}, \mathcal{A}_{b}^{(1 R)}$ and $\mathcal{A}_{t}^{(0)}$.

Sherpa [49]. At the level of the total cross section advancements have been made by first understanding the differences between results obtained in the two schemes refs. $[50,51]$ and then by consistently combining state-of-the-art $4 \mathrm{FS}$ and $5 \mathrm{FS}$ predictions in refs. [52-55].

The paper is organised as follows. In section 2 our computation is described in detail. We first discuss the various contributions to the $b \bar{b} H$ cross section (section 2.1), then introduce the HEFT approximation to determine the $y_{t}^{2}$ terms (section 2.2) and finally perform a comprehensive validation of the HEFT approximation for the $y_{t}^{2}$ cross section (section 2.3); phenomenological results are presented in section 3 - see in particular section 3.1 for the input parameters, section 3.2 for SM results, section 3.3 for how to obtain the best sensitivity to extract $y_{b}$ in the measurements, and section 3.4 for our analysis on NLO corrections to $g \rightarrow b \bar{b}$ splitting. We conclude in section 4 and collect relevant technical information in the appendices.

\section{Outline of the calculation}

\subsection{Coupling structure of the $b \bar{b} H$ cross section}

The leading contribution to the associated production of a Higgs boson with bottom quarks in the 4FS starts at $\mathcal{O}\left(\alpha_{s}^{2}\right)$ in QCD perturbation theory, and is mediated by the bottomquark Yukawa coupling. Hence, the coupling structure of the LO process is $y_{b}^{2} \alpha_{s}^{2}$. A sample Feynman diagram is shown in figure 1a. At the next order in $\alpha_{s}$ the typical one-loop (figure 1b) and real-emission (figure 1c) diagrams are included, and yield a contribution of $\mathcal{O}\left(y_{b}^{2} \alpha_{s}^{3}\right)$. At the same order in $\alpha_{s}$ additional one-loop diagrams appear featuring a closed top-quark loop which the Higgs boson couples to (figure 1d). These diagrams introduce for the first time a dependence on top-quark Yukawa coupling in the $b \bar{b} H$ cross section and lead to contributions of $\mathcal{O}\left(y_{b} y_{t} \alpha_{s}^{3}\right)$ through their interference with $y_{b}$ diagrams as shown in figure 1a. At the next order in $\alpha_{s}$, the square of these $y_{t}$ amplitudes yields a contribution that starts at $\mathcal{O}\left(y_{t}^{2} \alpha_{s}^{4}\right)$. Thus, it is suppressed by two powers of $\alpha_{s}$ with respect to the first non-zero contribution to $b \bar{b} H$ production of $\mathcal{O}\left(y_{b}^{2} \alpha_{s}^{2}\right)$ and could be formally considered a NNLO contribution. However, it is easy to understand that a naïve power counting just based on the single parameter $\alpha_{s}$ is not suitable for describing $b \bar{b} H$ production, since the strong hierarchy between the top-quark and the bottom-quark Yukawa couplings in the SM 


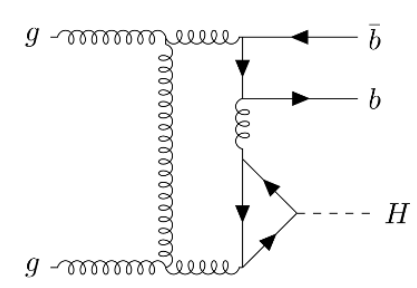

(a) $\mathcal{A}_{t}^{(1 V)}$

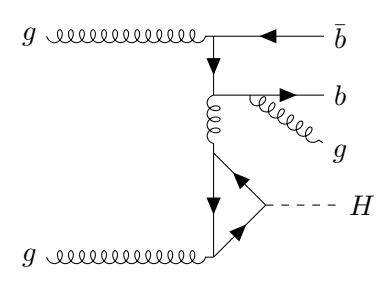

(b) $\mathcal{A}_{t}^{(1 R)}$

Figure 2. Examples of virtual, $\mathcal{A}_{t}^{(1 V)}$, and real emission, $\mathcal{A}_{t}^{(1 R)}$, diagrams contributing to associated $b \bar{b} H$ production at $\mathcal{O}\left(y_{t}^{2} \alpha_{s}^{5}\right)$, and $\mathcal{O}\left(y_{b} y_{t} \alpha_{s}^{4}\right)$ through their interference with $\mathcal{A}_{b}^{(0)}$ and $\mathcal{A}_{b}^{(1 R)}$.

is such that $y_{b}^{2} \alpha_{s}^{2}$ terms turn out to be of a similar size as the $y_{t}^{2} \alpha_{s}^{4}$ contributions. In this respect, one also expects that $\alpha_{s}$ corrections to the $y_{t}^{2}$ contributions might turn out to be important, which are of $\mathcal{O}\left(y_{t}^{2} \alpha_{s}^{5}\right)$ and formally part of the $\mathrm{N}^{3} \mathrm{LO}$ corrections with respect to the leading $\mathcal{O}\left(y_{b}^{2} \alpha_{s}^{2}\right)$ terms. They enter via virtual and real diagrams of the type shown in figure $2 \mathrm{a}$ and in figure $2 \mathrm{~b}$, respectively.

Collecting all relevant terms at different orders in $\alpha_{s}$, one can express the cross section as

$$
\begin{aligned}
\mathrm{d} \sigma \propto & \alpha_{s}^{2} y_{b}^{2}\left|\mathcal{A}_{b}^{(0)}\right|^{2} \\
& +\alpha_{s}^{3}\left[y_{b}^{2}\left(2 \operatorname{Re}\left(\mathcal{A}_{b}^{(0)} \mathcal{A}_{b}^{(1 V)}\right)+\int \mathrm{d} \Phi\left|\mathcal{A}_{b}^{(1 R)}\right|^{2}\right)+y_{b} y_{t} 2 \operatorname{Re}\left(\mathcal{A}_{b}^{(0)} \mathcal{A}_{t}^{(0)}\right)\right] \\
& +\alpha_{s}^{4}\left[y_{t} y_{b} 2 \operatorname{Re}\left(\mathcal{A}_{b}^{(0)} \mathcal{A}_{t}^{(1 V)}+\mathcal{A}_{b}^{(1 V)} \mathcal{A}_{t}^{(0)}+\int \mathrm{d} \Phi \mathcal{A}_{b}^{(1 R)} \mathcal{A}_{t}^{(1 R)}\right)+y_{t}^{2}\left|\mathcal{A}_{t}^{(0)}\right|^{2}\right] \\
& +\alpha_{s}^{5} y_{t}^{2}\left[2 \operatorname{Re}\left(\mathcal{A}_{t}^{(1 V)} \mathcal{A}_{t}^{(0)}\right)+\int \mathrm{d} \Phi\left|\mathcal{A}_{t}^{(1 R)}\right|^{2}\right] \\
& +\mathcal{O}\left(\alpha_{s}^{4} y_{b}^{2}\right)+\mathcal{O}\left(\alpha_{s}^{5} y_{b} y_{t}\right)+\mathcal{O}\left(\alpha_{s}^{6} y_{t}^{2}\right),
\end{aligned}
$$

where the $\mathcal{A}_{q}^{(i)}$ amplitudes are introduced with the respective sample diagrams in figures 1-2, and $\mathrm{d} \Phi$ denotes the appropriate phase space of the extra real emission with all relevant factors in each case. An equivalent, yet more appropriate and transparent way of organising the computation above is to consider a double coupling expansion in terms of $y_{b}$ and $y_{t}$ and then to systematically include $\alpha_{s}$ corrections to each of these terms. ${ }^{2}$ Up to NLO, the cross section can be written as

$$
\mathrm{d} \sigma=y_{b}^{2} \alpha_{s}^{2}\left(\Delta_{y_{b}^{2}}^{(0)}+\alpha_{s} \Delta_{y_{b}^{2}}^{(1)}\right)+y_{t} y_{b} \alpha_{s}^{3}\left(\Delta_{y_{b} y_{t}}^{(0)}+\alpha_{s} \Delta_{y_{b} y_{t}}^{(1)}\right)+y_{t}^{2} \alpha_{s}^{4}\left(\Delta_{y_{t}^{2}}^{(0)}+\alpha_{s} \Delta_{y_{t}^{2}}^{(1)}\right) .
$$

It is trivial to see that eq. (2.1) and eq. (2.2) feature exactly the same terms. In this formulation QCD corrections to $y_{b}^{2}, y_{b} y_{t}$ and $y_{t}^{2}$ terms, the $\Delta_{x}^{(i)}$ contributions, are manifestly gauge invariant and can be calculated independently of each other at LO and NLO. All the coefficients up to $\mathcal{O}\left(\alpha_{s}^{3}\right)\left(\Delta_{y_{b}^{2}}^{(0)}, \Delta_{y_{b}^{2}}^{(1)}\right.$, and $\left.\Delta_{y_{b} y_{t}}^{(0)}\right)$ were determined and studied already

\footnotetext{
${ }^{2}$ This is possible because QCD corrections do not induce any other coupling combinations on top of $y_{b}^{2}$, $y_{t}^{2}, y_{b} y_{t}$.
} 
in ref. [30]. Our focus here is therefore on the calculation of the contributions involving $y_{t}$ in the 4 FS, i.e., $\Delta_{y_{b} y_{t}}^{(1)}, \Delta_{y_{t}^{2}}^{(0)}$, and $\Delta_{y_{t}^{2}}^{(1)}{ }^{3}$

\subsection{HEFT approximation in $b \bar{b} H$ production}

NLO corrections to the contributions proportional to the top-quark Yukawa coupling require the computation of two-loop $2 \rightarrow 3$ amplitudes with internal massive fermion lines, see figure 2a. The evaluation of such diagrams is beyond current technology. Hence, in this section, we introduce the heavy top-mass approximation that can be employed for the computation of these amplitudes, and we rearrange the SM cross section discussed in section 2.1 in the HEFT.

In this effective theory, the top quark is integrated out and yields effective point-like interactions between the Higgs boson and gluons, described by the effective Lagrangian

$$
\mathcal{L}=-\frac{1}{4} C_{1} H G_{\mu \nu}^{a} G^{a, \mu \nu},
$$

where $H$ denotes the field associated to the physical Higgs boson, $v$ is the vacuum expectation value of the Higgs field, $G_{\mu \nu}^{a}$ is the gluon field strength tensor and $C_{1}$ is the Wilson coefficient that can be expressed in terms of SM parameters. The HEFT Lagrangian yields a point-like interaction for both $H g g$ and $H g g g$ vertices. At leading order in the strong coupling, we have

$$
C_{1}=-\frac{1}{v}\left[y_{t} \frac{v}{\sqrt{2} m_{t}}\right]\left(\frac{\alpha_{s}}{3 \pi}+\mathcal{O}\left(\alpha_{s}^{2}\right)\right)
$$

where the dependence on the top Yukawa coupling, which is explicit in the SM, is cancelled by the power supression of the loop integral, making the term in brackets exactly equal to 1. By matching the amplitude for the process $H \rightarrow g g$ in the HEFT and the SM at higher orders in perturbation theory, the expansion of $C_{1}$ in $\alpha_{s}$ can be determined [58], which will then depend on the renormalization schemes adopted in the HEFT and in the SM. We discuss the details of the renormalization procedure in appendix B.

The HEFT approximation has been used successfully to compute a number of observables in the Higgs sector, with the gluon-fusion cross section through $\mathrm{N}^{3} \mathrm{LO}$ as the most notable example [57, 59,60]. By substituting top loops with a point-like coupling, the HEFT allows for significant simplifications of Higgs-related observables at the price of a limited range of applicability: the approximation is expected to break down when one of the scales appearing in the process, and in particular in the massive loop integrals, becomes comparable with the top-quark mass. The case at hand corresponds to $H+$ jet $(H+g)$ production with $g \rightarrow b \bar{b}$ splitting either in the initial or in the final state. It has been shown that the HEFT provides an excellent approximation in that case as long as the scales of the process remain moderate [61, 62], for example as long as the Higgs transverse momentum $\left(p_{H}^{T}\right)$ is below $\sim 150 \mathrm{GeV}$. In section 2.3 , we provide a detailed assessment of the goodness

\footnotetext{
${ }^{3}$ The contributions $\Delta_{y_{t}^{2}}^{(0)}\left(\Delta_{y_{t}^{2}}^{(1)}\right)$ are implicitly included in the computation of gluon-gluon fusion at NNLO $\left(\mathrm{N}^{3} \mathrm{LO}\right)$ in the 5FS $[56,57]$. These calculations, however, cannot provide information on final states specifically containing $b$ quarks.
} 


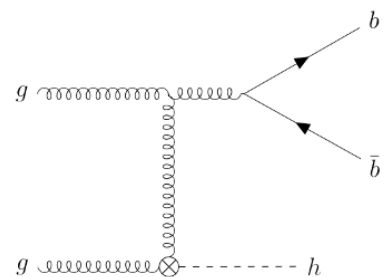

(a) $\hat{\mathcal{A}}_{t}^{(0)}$

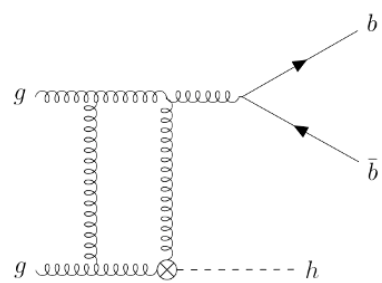

(b) $\hat{\mathcal{A}}_{t}^{(1 V)}$

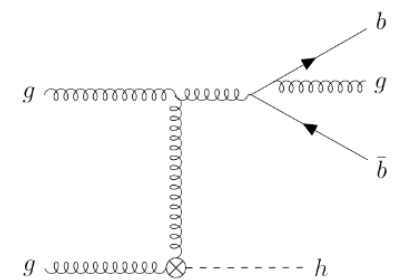

(c) $\hat{\mathcal{A}}_{t}^{(1 R)}$

Figure 3. Examples of Born-level, virtual and real-emission diagrams for the $y_{t}^{2}$ contribution to $b \bar{b} H$ production in the heavy-top quark approximation.

of the heavy top-mass approximation. As we will show, the heavy-top mass approximation works extremely well (with differences from the full computation below 10\%) as long as the probed momentum scales (Higgs or leading b-jet transverse momentum, or invariant mass of the b-jet pair) do not exceed $200 \mathrm{GeV}$.

Working in the HEFT allows us to avoid the computation of the highly complicated amplitudes in figure 1d and figure 2, and evaluate instead the diagrams shown in figure 3, which have a much lower complexity, being at most at the one-loop level. In addition, the HEFT has been implemented in a Universal Feynrules Output (UFO) model [63, 64], and this calculation can be performed using existing automated Monte Carlo tools. Nonetheless, present implementations neglect power-suppressed corrections to SM parameters generated by the heavy-top mass approximation, which play a crucial role in the case at hand. In particular the bottom-quark Yukawa coupling must be corrected in the following way:

$$
y_{b}^{\mathrm{HEFT}}=y_{b}+y_{t} \alpha_{s}^{2} \frac{m_{b}}{m_{t}} \delta y_{b},
$$

which generates additional terms of $\mathcal{O}\left(y_{b} y_{t} \alpha_{s}^{4}\right)$ and $\mathcal{O}\left(y_{t}^{2} \alpha_{s}^{5}\right)$ entering the $b \bar{b} H$ cross section at the perturbative order we are interested in. The exact expression for $\delta y_{b}$ can be obtained from eq. (2.7). As a result, we insert $y_{b} \rightarrow y_{b}^{\mathrm{HEFT}}$ into eq. (2.2) to yield the $b \bar{b} H$ cross section in the HEFT and rearrange it as follows:

$$
\begin{aligned}
\mathrm{d} \sigma^{\mathrm{HEFT}}= & y_{b}^{2} \alpha_{s}^{2}\left(\Delta_{y_{b}^{2}}^{(0)}+\alpha_{s} \Delta_{y_{b}^{2}}^{(1)}\right) \\
& +y_{t} y_{b} \alpha_{s}^{3}\left(\hat{\Delta}_{y_{b} y_{t}}^{(0)}+\alpha_{s} \hat{\Delta}_{y_{b} y_{t}}^{(1)}+2 \alpha_{s} \frac{m_{b}}{m_{t}} \delta y_{b} \Delta_{y_{b}^{2}}^{(0)}\right) \\
& +y_{t}^{2} \alpha_{s}^{4}\left(\hat{\Delta}_{y_{t}^{2}}^{(0)}+\alpha_{s} \hat{\Delta}_{y_{t}^{2}}^{(1)}+\alpha_{s} \frac{m_{b}}{m_{t}} \delta y_{b} \hat{\Delta}_{y_{b} y_{t}}^{(0)}\right)
\end{aligned}
$$

where top-quark loops have been replaced by the HEFT contact interaction in quantities with a hat. ${ }^{4}$ In this cross section, the only contribution that could not be directly calculated

\footnotetext{
${ }^{4}$ In practice we do not replace loops explicitly in the SM calculation: our UFO model contains all gaugeinvariant leading power interactions of the HEFT and we generate the HEFT amplitude independently using MADGRAPH5_AMC@NLO [65]. The expressions for the $\hat{\Delta}$ terms contains all tree-level, real-emission and virtual diagrams that are relevant after replacing $C_{1}$ by its matched expression to order $\mathcal{O}\left(y_{t} \alpha_{s}^{2}\right)$. We articifically separate the insertion of the matched expression for the bottom Yukawa in eq. (2.6) to attract the reader's attention to the effect of this power suppressed correction, which would be missed if one only replaced top loops by contact operators - even when consistently including higher orders of the matched Wilson coefficient.
} 
using automated tools is the power-suppressed bottom-quark Yukawa correction. This was derived using a low-energy theorem, at $\mathcal{O}\left(\alpha_{s}^{2}\right)$ in refs. [66, 67], which is the order needed for this calculation, and further improved to $\mathcal{O}\left(\alpha_{s}^{4}\right)$ in ref. [68]. We rederive the $\mathcal{O}\left(\alpha_{s}^{2}\right)$ coefficient in the appendix $\mathrm{C}$ by an explicit two-loop matching calculation and find:

$$
y_{b}^{\mathrm{HEFT}}=y_{b}^{\mathrm{SM}}+y_{t}\left(\frac{\alpha_{s}}{\pi}\right)^{2} \frac{m_{b}}{m_{t}} C_{F}\left(\frac{5}{24}-\frac{1}{4} \log \left(\frac{\mu_{R}^{2}}{m_{t}^{2}}\right)\right),
$$

in agreement with the existing literature, where $\alpha_{s}$ and $y_{b}^{\mathrm{SM}}$ are understood to be renormalised in the $\overline{\mathrm{MS}}$ scheme at a scale $\mu_{R}$, while $m_{b}, m_{t}$ and $y_{t}$ are renormalised on-shell. Note that the renormalization scheme of SM parameters affects the matching coefficient $\delta y_{b}$ only at higher orders, neglected in the present calculation.

We have implemented by hand this modification in the HEFT model at NLO. This enables a complete calculation of the QCD corrections $\hat{\Delta}_{y_{t}^{2}}^{(1)}$ and $\hat{\Delta}_{y_{b} y_{t}}^{(1)}$ in the heavy-top mass approximation in a fully automated way. We therefore can employ MADGRAPH5_AMC@NLO [65] to perform the calculation of the $b \bar{b} H$ cross section in the $4 \mathrm{FS}$ at parton level. We use the recently-released version capable of computing a mixed-coupling expansion [69] of the cross section in order to compute all six contributions $\left(y_{b}^{2}, y_{b} y_{t}\right.$ and $y_{t}^{2}$ both at $\mathrm{LO}$ and $\left.\mathrm{NLO}\right)$ with the appropriate $\overline{\mathrm{MS}}$ renormalisation of $y_{b}$ simultaneously. ${ }^{5}$

Besides computing eq. (2.6) in the HEFT, we also calculate the LO $y_{t}^{2}$ contributions in the full theory in order to rescale the $y_{t}^{2}$ contributions and to provide the best approximation of the $b \bar{b} H$ cross section in eq. (2.2). We refer to this approach as the Born-improved HEFT (BI-HEFT) in the following:

$$
\sigma_{y_{t}^{2}}^{\mathrm{BI}-\mathrm{HEFT}} \equiv \sigma_{y_{t}^{2}}^{\mathrm{HEFT}} \times \frac{\sigma_{y_{t}^{2}}^{\mathrm{SM}, \mathrm{LO}}}{\sigma_{y_{t}^{2}}^{\mathrm{HEFT}, \mathrm{LO}}} .
$$

For differential distributions, eq. (2.8) is applied bin-by-bin.

\subsection{Assessment of the HEFT approximation}

In this section we assess the accuracy of the heavy-top quark approximation. To this end, we compare the LO $y_{t}^{2}$ cross section $\sigma_{y_{t}^{2}}^{\mathrm{SM}}$ against its approximation in the HEFT $\sigma_{y_{t}^{2}}^{\mathrm{HEFT}}$. We use the same input parameters as for our phenomenological results in section 3 , and refer to section 3.1 for details. We perform a validation for both the inclusive cross section and differential distributions. Since the topology of the process at LO is very similar to that of the $H$ +jet process, we expect the HEFT to provide a good description in the relevant phase-space regions, in particular concerning the shapes of distributions. We stress again that in our best prediction, the BI-HEFT, we use the HEFT only to determine the radiative corrections in terms of the NLO $K$-factor. Total cross section and kinematic distributions, obtained in the HEFT, are reweighted (bin-by-bin) by a factor equal to the ratio between the full theory and the HEFT, both evaluated at LO. This has been shown to be an

\footnotetext{
${ }^{5} \mathrm{~A}$ similar computation was performed in the context of charged-Higgs production in the intermediatemass range [70].
} 
excellent approximation for $H+$ jet production as long as the relevant scales do not become too large $[61,62]$.

We start by reporting the result for the inclusive cross section:

$$
\sigma_{y_{t}^{2}}^{\mathrm{SM}}=0.375 \mathrm{pb}, \quad \sigma_{y_{t}^{2}}^{\mathrm{HEFT}}=0.358 \mathrm{pb} .
$$

The results lie within $5 \%$ of each other. Considering that the perturbative uncertainties are one order of magnitude larger, we conclude that the inclusive cross section is well described by the heavy-top quark approximation. Furthermore, the accuracy of the BI-HEFT result can be assumed to be considerably better than this value, since top-mass effects are included at LO by the rescaling in eq. (2.8). As in the case of $H+$ jet production, the dominant configurations are with the Higgs at low transverse momentum, which explains the quality of the approximation.

Let us now turn to differential cross sections in figure 4. The main frame shows the SM (blue dash-dotted) and HEFT (green dotted) predictions. The lower inset shows their bin-by-bin ratios. The first three plots, figures $4 \mathrm{a}-4 \mathrm{c}$ feature the transverse-momentum spectra of the Higgs boson, the leading and the subleading $b$ jet respectively. As expected, we find that the HEFT provides a good description of the SM result, especially in terms of shapes. Only at large transverse momentum the two curves start deviating with the HEFT result becoming harder. This happens after transverse momenta of $\sim 200 \mathrm{GeV}$ for the Higgs and the leading $b$ jet, and a bit earlier for the second-hardest $b$ jet.

The Higgs rapidity distribution in figure $4 \mathrm{~d}$ is hardly affected by the HEFT approximation, with the HEFT/SM ratio being essentially flat. Also for the invariant mass of the two $b$ jets in figure 4e, the heavy-top quark result provides a good description as long as $M(b b) \lesssim 200 \mathrm{GeV}$. Finally, for the separation in the $\eta-\phi$ plane between the two $b$ jets, shown in figure $4 \mathrm{f}$, the agreement between HEFT and SM is very good up to $\Delta R=4$. Above this value, the distribution is dominated by large invariant-mass pairs, and the HEFT/SM ratio follows what happens for the invariant-mass distribution.

Overall, the heavy-top quark approximation used in the HEFT results works extremely well for this process over a large fraction of the phase space and in particular where the majority of events are produced. For the goals of our study, it is especially important to verify that the comparison of the angular separation of the $b$ jets and of their invariant mass is well reproduced, as it indicates that we can safely explore the regime in which the two bottom jets merge into a single one. This regime is particularly interesting to study for the $y_{t}^{2}$ terms as we will see in section 3. Furthermore, there is a reasonable range of $b$-jet transverse momentum where the process is correctly described, so that we can trust the prediction to study the impact of $b$-jet requirements on the relative importance of the $y_{b}^{2}$ and $y_{t}^{2}$ contributions. It should be noted, however, that in the two $b$-jet configuration, the HEFT prediction is rather poor over a larger range of transverse momenta for the subleading $b$ jet. Nevertheless, this is not expected to have an impact on our phenomenological study in the upcoming section. 


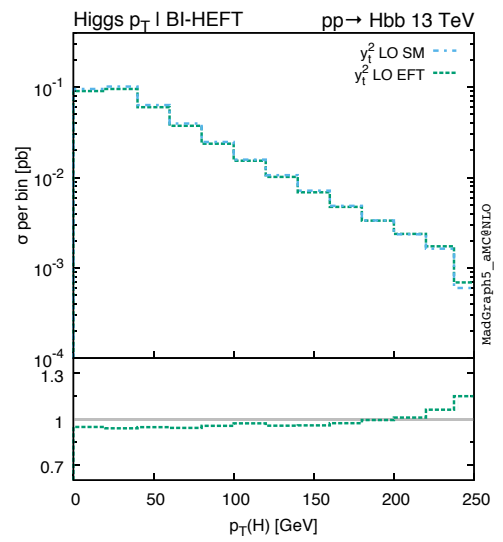

(a)

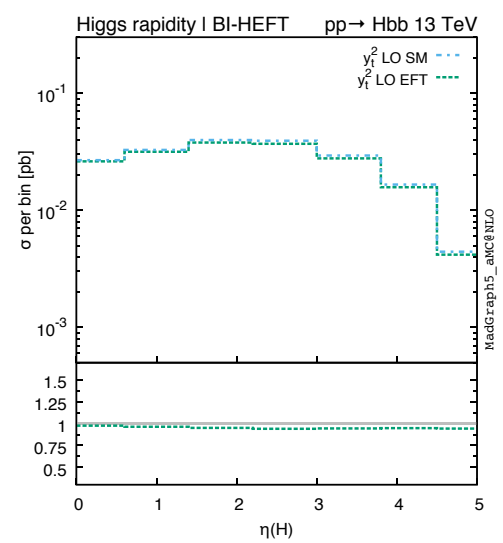

(d)

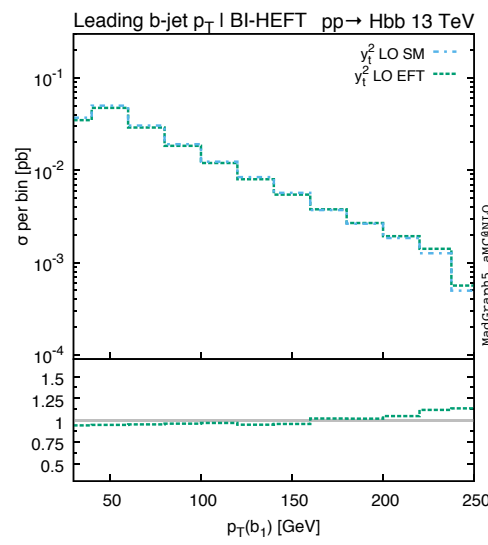

(b)

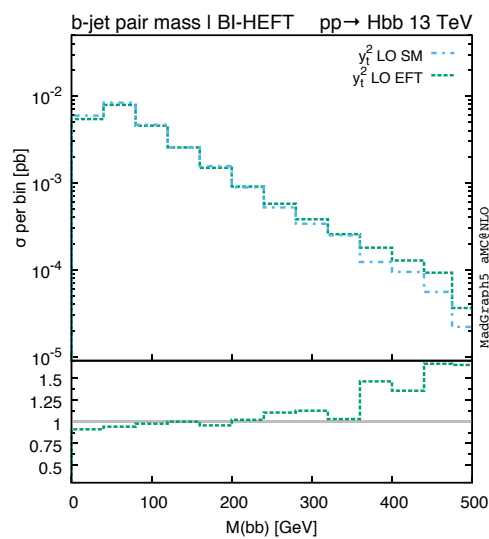

(e)

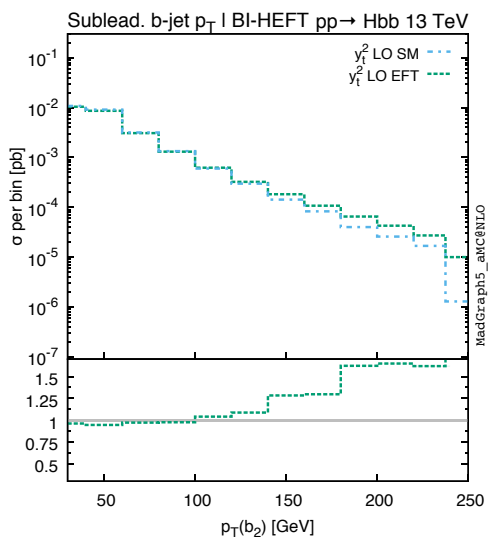

(c)

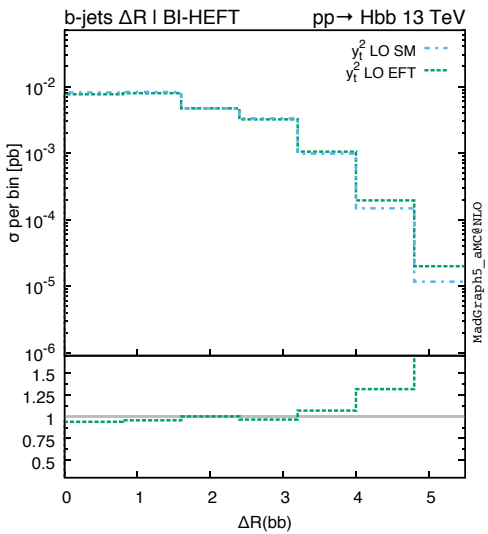

(f)

Figure 4. Comparison of LO predictions in the SM and the HEFT for various observables: the transverse-momentum of the Higgs boson (4a), of the leading (4b), and of the subleading $b$ jet (4c), the rapidity of the Higgs boson (4d), the invariant mass of the $b$-jet pair (4e), and their distance in the $\eta-\phi$ plane (4f); the lower insets show the ratio of the two predictions.

\section{Phenomenological results}

In this section we present differential results for $b \bar{b} H$ production at the $13 \mathrm{TeV}$ LHC including all contributions proportional to $y_{b}^{2}, y_{b} y_{t}$, and $y_{t}^{2}$ at NLO QCD, see eq. (2.2). We analyze the importance of radiative corrections and the relative size of the three contributions. Although we work in the SM, thanks to the separation of the cross section by the Yukawa coupling structure, our predictions are directly applicable to 2HDM-type extensions of the SM (for $b \bar{b} \phi$ with a neutral Higgs boson $\phi \in\{h, H, A\}$ ) by an appropriate rescaling of the top and bottom-quark Yukawa. Even for the MSSM such rescaling has been shown to be an excellent approximation of the complete result [71, 72].

\subsection{Input parameters}

Our predictions are obtained in the four-flavour scheme throughout. We use the corresponding $n_{f}=4$ NNPDF 3.1 [73] sets of parton densities at NLO with the corresponding 
running and $\alpha_{s}$ values. ${ }^{6}$ The central values of the renormalisation $\left(\mu_{R}\right)$ and factorisation $\left(\mu_{F}\right)$ scales are set on an event-to-event basis to

$$
\mu_{R}=\mu_{F}=H_{T} / 4=\frac{1}{4} \sum_{i} \sqrt{\left(p_{i}^{T}\right)^{2}+m_{i}^{2}}
$$

where the index $i$ runs over all the final-state particles, possibly including the extra parton from the real emission. Scale uncertainties are computed without extra runs using a reweighting technique [75], and correspond to independent (nine-point) variations in the range $H_{T} / 8 \leq \mu_{R}, \mu_{F} \leq H_{T} / 2$. Internal masses are set to their on-shell values $m_{H}=125 \mathrm{GeV}, m_{t}=172.5 \mathrm{GeV}$ and $m_{b}=4.92 \mathrm{GeV}$. The top-quark Yukawa is renormalised on-shell; for the bottom-quark Yukawa, instead, we compute $m_{b}\left(\mu_{R}\right)$ by adopting the $\overline{\mathrm{MS}}$ scheme, with a four-loop evolution $[76,77]$ from $m_{b}\left(m_{b}\right)=4.18 \mathrm{GeV}$ up to the central value of the renormalisation scale, and two-loop running for the scale variations, as recommended by the LHC Higgs cross section working group [78].

Jets are reconstructed with the anti- $k_{T}$ algorithm [79], as implemented in FASTJET [80], with a jet radius of $R=0.4$, and subject to the condition $p_{j}^{T}>30 \mathrm{GeV}$ and $\eta_{j}<2.5$. Results with a larger jet radius, $R=1$, are available in appendix A. Bottom-quark flavoured jets ( $b$ jets) are defined to include at least one bottom quark among the jet constituents. A $b$ jet containing a pair of bottom quarks is denoted as a $b b$ jet. Within a fixed-order computation, we will use the word $B$ hadrons to identify bottom quarks (the notation $B$ will refer to bottom quarks, while the notation $b$ to bottom-tagged jets). Bottom-quark observables are infrared safe owing to the finite bottom-quark mass in the $4 \mathrm{FS}$. At variance with the case of $b$ jets, no cut is imposed on $B$ hadrons.

\subsection{Predictions for $b \bar{b} H$ production in the SM}

We start by discussing integrated cross sections in table 1, both fully inclusive and within cuts. As far as the latter are concerned, we have considered various possibilities: the requirement that there be at least one or two $b$ jet(s); that there be at least one jet containing a pair of bottom quarks ( $b b$ jets); and that the transverse momentum of the Higgs boson be larger than $50 \mathrm{GeV}, 100 \mathrm{GeV}$, and $150 \mathrm{GeV}$ (boosted scenarios), for simplicity without any requirement on $b$ jets. The residual scale uncertainties are computed by varying the scales as indicated in section 3.1. We present separately the results for terms proportional to $y_{b}^{2}, y_{t}^{2}$, and $y_{b} y_{t}$. The $y_{t}^{2}$ contributions are provided in two approximations: using the HEFT, on the one hand, and our BI-HEFT prediction, computed by rescaling the HEFT result at NLO by the LO evaluated in the full theory, on the other hand. For completeness, we also quote the BI-HEFT prediction for the sum of all individual contributions. Besides LO and NLO of the cross sections we also provide the NLO/LO $K$-factor to assess the importance of QCD corrections. Inside the bracket after the LO and NLO cross sections we quote the acceptance of the respective scenario, defined as the ratio of the cross section within cuts divided by the inclusive one. We refrain from quoting the

\footnotetext{
${ }^{6}$ More precisely, NNPDF31_nlo_as_0118_nf_4 (lhaid=320500 in LHAPDF6 [74]) corresponding to $\alpha_{s}\left(m_{Z}\right)=0.118$.
} 


\begin{tabular}{|c|c|c|c|c|}
\hline & & LO (acceptance) & NLO (acceptance) & $K$ \\
\hline \multirow{5}{*}{ inclusive } & $y_{t \text { HEFT }}^{2}$ & $3.58 \cdot 10^{-1}{ }_{-39 \%}^{+74 \%}(100 \%)$ & $8.80 \cdot 10^{-1}{ }_{-31 \%}^{+47 \%}(100 \%)$ & 2.5 \\
\hline & $y_{t \text { BI-HEFT }}^{2}$ & $3.75 \cdot 10^{-1}{ }_{-39 \%}^{+74 \%}(100 \%)$ & $9.22 \cdot 10^{-1}{ }_{-31 \%}^{+47 \%}(100 \%)$ & 2.5 \\
\hline & $y_{b} y_{t}$ & $-3.82 \cdot 10^{-2}{ }_{-36 \%}^{+65 \%}(\square)$ & $-7.37 \cdot 10^{-2}{ }_{-27 \%}^{+39 \%}(\square)$ & 1.9 \\
\hline & $y_{b}^{2}$ & $2.63 \cdot 10^{-1}{ }_{-34 \%}^{+57 \%}(100 \%)$ & $4.05 \cdot 10^{-1}{ }_{-21 \%}^{+21 \%}(100 \%)$ & 1.5 \\
\hline & $y_{b}^{2}+y_{b} y_{t}+y_{t \text { BI-HEFT }}^{2}$ & $6.00 \cdot 10^{-1}{ }_{-37 \%}^{+67 \%}(100 \%)$ & $1.25 \cdot 10^{0} \quad{ }_{-28 \%}^{+38 \%}(100 \%)$ & 2.1 \\
\hline \multirow{5}{*}{$\geq 1 b$} & $y_{t}^{2}$ HEFT & $1.70 \cdot 10^{-1}{ }_{-39 \%}^{+72 \%}(47 \%)$ & $3.67 \cdot 10^{-1}{ }_{-29 \%}^{+39 \%}(42 \%)$ & 2.2 \\
\hline & $y_{t}^{2} \mathrm{BI}$-HEFT & $1.76 \cdot 10^{-1}{ }_{-39 \%}^{+72 \%}(47 \%)$ & $3.81 \cdot 10^{-1}{ }_{-29 \%}^{+39 \%}(41 \%)$ & 2.2 \\
\hline & $y_{b} y_{t}$ & $-1.15 \cdot 10^{-2}{ }_{-35 \%}^{+62 \%}(\square)$ & $-1.63 \cdot 10^{-2}{ }_{-19 \%}^{+16 \%}(\square)$ & 1.4 \\
\hline & $y_{b}^{2}$ & $6.02 \cdot 10^{-2}{ }_{-31 \%}^{+52 \%}(23 \%)$ & $8.49 \cdot 10^{-2}{ }_{-16 \%}^{+13 \%}(21 \%)$ & 1.4 \\
\hline & $y_{b}^{2}+y_{b} y_{t}+y_{t}^{2} \mathrm{BI}-\mathrm{HEFT}$ & $2.25 \cdot 10^{-1}{ }_{-37 \%}^{+67 \%}(37 \%)$ & $4.50 \cdot 10^{-1}{ }_{-27 \%}^{+35 \%}(36 \%)$ & 2.0 \\
\hline \multirow{5}{*}{$\geq 2 b$} & $y_{t \mathrm{HEFT}}^{2}$ & $2.48 \cdot 10^{-2}{ }_{-39 \%}^{+72 \%}(6.9 \%)$ & $4.86 \cdot 10^{-2}{ }_{-27 \%}^{+33 \%}(5.5 \%)$ & 2.0 \\
\hline & $y_{t \text { BI-HEFT }}^{2}$ & $2.56 \cdot 10^{-2}{ }_{-39 \%}^{+72 \%}(6.8 \%)$ & $5.02 \cdot 10^{-2}{ }_{-27 \%}^{+33 \%}(5.4 \%)$ & 2.0 \\
\hline & $y_{b} y_{t}$ & $-6.95 \cdot 10^{-4}{ }_{-35 \%}^{+62 \%}(\square)$ & $-5.24 \cdot 10^{-4}{ }_{-53 \%}^{+5 \%}(-)$ & 0.8 \\
\hline & $y_{b}^{2}$ & $5.07 \cdot 10^{-3}{ }_{-31 \%}^{+51 \%}(1.9 \%)$ & $5.92 \cdot 10^{-3}{ }_{-12 \%}^{+1 \%}(1.5 \%)$ & 1.2 \\
\hline & $y_{b}^{2}+y_{b} y_{t}+y_{t \mathrm{BI}-\mathrm{HEFT}}^{2}$ & $3.00 \cdot 10^{-2}{ }_{-38 \%}^{+69 \%}(5.0 \%)$ & $5.56 \cdot 10^{-2}{ }_{-26 \%}^{+30 \%}(4.4 \%)$ & 1.9 \\
\hline \multirow{5}{*}{$\geq 1 b b$} & $y_{t \mathrm{HEFT}}^{2}$ & $3.84 \cdot 10^{-2}{ }_{-38 \%}^{+70 \%}(11 \%)$ & $7.86 \cdot 10^{-2}{ }_{-28 \%}^{+36 \%}(8.9 \%)$ & 2.0 \\
\hline & $y_{t \mathrm{BI}-\mathrm{HEFT}}^{2}$ & $4.12 \cdot 10^{-2}{ }_{-38 \%}^{+70 \%}(11 \%)$ & $8.43 \cdot 10^{-2}{ }_{-28 \%}^{+36 \%}(9.1 \%)$ & 2.0 \\
\hline & $y_{b} y_{t}$ & $-7.91 \cdot 10^{-5}{ }_{-45 \%}^{+89 \%}(\square)$ & $2.02 \cdot 10^{-4}{ }_{-54 \%}^{+132 \%}(\square)$ & -2.5 \\
\hline & $y_{b}^{2}$ & $3.37 \cdot 10^{-4}{ }_{-34 \%}^{+57 \%}(0.1 \%)$ & $2.53 \cdot 10^{-4}{ }_{-48 \%}^{+4 \%}(0.1 \%)$ & 0.7 \\
\hline & $y_{b}^{2}+y_{b} y_{t}+y_{t}^{2} \mathrm{BI}$-HEFT & $4.15 \cdot 10^{-2}{ }_{-38 \%}^{+70 \%}(6.9 \%)$ & $8.48 \cdot 10_{-28 \%}^{-2}{ }_{-26 \%}^{+36 \%}(6.8 \%)$ & 2.0 \\
\hline \multirow{5}{*}{$p_{H}^{T}>50 \mathrm{GeV}$} & $y_{t \text { HEFT }}^{2}$ & $1.38 \cdot 10^{-1}{ }_{-39 \%}^{+73 \%}(39 \%)$ & $3.77 \cdot 10^{-1}{ }_{-33 \%}^{+52 \%}(43 \%)$ & 2.7 \\
\hline & $y_{t \text { BI-HEFT }}^{2}$ & $1.42 \cdot 10^{-1}{ }_{-39 \%}^{+73 \%}(38 \%)$ & $3.87 \cdot 10^{-1}{ }_{-33 \%}^{+52 \%}(42 \%)$ & 2.7 \\
\hline & $y_{b} y_{t}$ & $-7.43 \cdot 10^{-3}{ }_{-35 \%}^{+62 \%}(\square)$ & $-9.66 \cdot 10^{-3}{ }_{-17 \%}^{+10 \%}(\square)$ & 1.3 \\
\hline & $y_{b}^{2}$ & $3.20 \cdot 10^{-2}{ }_{-32 \%}^{+53 \%}(12 \%)$ & $5.54 \cdot 10^{-2}{ }_{-21 \%}^{+24 \%}(14 \%)$ & 1.7 \\
\hline & $y_{b}^{2}+y_{b} y_{t}+y_{t \mathrm{BI}-\mathrm{HEFT}}^{2}$ & $1.66 \cdot 10^{-1}{ }_{-38 \%}^{+70 \%}(28 \%)$ & $4.33 \cdot 10^{-1}{ }_{-32 \%}^{+49 \%}(35 \%)$ & 2.6 \\
\hline \multirow{5}{*}{$p_{H}^{T}>100 \mathrm{GeV}$} & $y_{t}^{2}$ HEFT & $5.03 \cdot 10^{-2}{ }_{-39 \%}^{+73 \%}(14 \%)$ & $1.43 \cdot 10^{-1}{ }_{-33 \%}^{+53 \%}(16 \%)$ & 2.8 \\
\hline & $y_{t \mathrm{BI}-\mathrm{HEFT}}^{2}$ & $4.98 \cdot 10^{-2}{ }_{-39 \%}^{+73 \%}(13 \%)$ & $1.41 \cdot 10^{-1}{ }_{-33 \%}^{+53 \%}(15 \%)$ & 2.8 \\
\hline & $y_{b} y_{t}$ & $-1.35 \cdot 10^{-3}{ }_{-36 \%}^{+63 \%}(\square)$ & $-1.20 \cdot 10^{-3}{ }_{-32 \%}^{+2 \%}(\square)$ & 0.9 \\
\hline & $y_{b}^{2}$ & $5.65 \cdot 10^{-3}{ }_{-33 \%}^{+54 \%}(2.1 \%)$ & $9.86 \cdot 10^{-3}{ }_{-21 \%}^{+24 \%}(2.4 \%)$ & 1.7 \\
\hline & $y_{b}^{2}+y_{b} y_{t}+y_{t}^{2}$ BI-HEFT & $5.42 \cdot 10_{-39 \%}^{-2}{ }_{-32 \%}^{+72 \%}(9.0 \%)$ & $1.50 \cdot 10^{-1}{ }_{-33 \%}^{+51 \%}(12 \%)$ & 2.8 \\
\hline \multirow{5}{*}{$p_{H}^{T}>150 \mathrm{GeV}$} & $y_{t \text { HEFT }}^{2}$ & $2.10 \cdot 10^{-2}{ }_{-39 \%}^{+74 \%}(5.9 \%)$ & $6.16 \cdot 10^{-2}{ }_{-33 \%}^{+53 \%}(7.0 \%)$ & 2.9 \\
\hline & $y_{t \mathrm{BI}-\mathrm{HEFT}}^{2}$ & $1.95 \cdot 10^{-2}{ }_{-39 \%}^{+74 \%}(5.2 \%)$ & $5.73 \cdot 10^{-2}{ }_{-33 \%}^{+53 \%}(6.2 \%)$ & 2.9 \\
\hline & $y_{b} y_{t}$ & $-3.18 \cdot 10^{-4}{ }_{-36 \%}^{+64 \%}(\square)$ & $-1.97 \cdot 10^{-4}{ }_{-84 \%}^{+11 \%}(\square)$ & 0.6 \\
\hline & $y_{b}^{2}$ & $1.40 \cdot 10^{-3}{ }_{-33 \%}^{+55 \%}(0.5 \%)$ & $2.51 \cdot 10^{-3}{ }_{-22 \%}^{+25 \%}(0.6 \%)$ & 1.8 \\
\hline & $y_{b}^{2}+y_{b} y_{t}+y_{t \mathrm{BI}-\mathrm{HEFT}}^{2}$ & $2.06 \cdot 10^{-2}{ }_{-39 \%}^{+73 \%}(3.4 \%)$ & $5.96 \cdot 10^{-2}{ }_{-33 \%}^{+53 \%}(4.8 \%)$ & 2.9 \\
\hline
\end{tabular}

Table 1. Cross sections (in pb) for different $b$-jet multiplicities or minimum $p_{H}^{T}$ cuts. 
acceptance for the $y_{b} y_{t}$ interference terms since this quantity is meaningless on its own. The conclusions that can be drawn from the table are the following:

- Already at LO the $y_{t}^{2}$ terms yield a significant contribution to the SM $b \bar{b} H$ cross section. Due to sizable QCD corrections to the $y_{t}^{2}$ terms $(K \approx 2.5)$, the inclusive NLO cross section is a factor of three larger after including the loop-induced gluon-fusion component than when considering only $y_{b}^{2}$ contributions. Hence, the $b \bar{b} H$ cross section in the SM is substantially larger than generally assumed from $y_{b}^{2}$ computations, which could make its observation much easier. At the same time, however, the sensitivity to the extraction of the bottom-quark Yukawa coupling is diminished. Below, we discuss how suitable phase-space cuts can be used to enhance the $y_{b}^{2}$ over the $y_{t}^{2}$ contributions and to retain sensitivity to the extraction of $y_{b}$.

- The relative size of $y_{t}^{2}$ contributions further increases when considering the various scenarios with additional phase-space cuts. The reason is that the loop-induced gluon-fusion component generates harder ( $b$-)jet activity and the cuts favour hard configurations. For example, tagging one $b$ jet has the effect of decreasing the $y_{b}^{2}$ NLO cross section by $-79 \%$, while for $y_{t}^{2}$ it is only $-59 \%$, and the $y_{t}^{2}$ NLO cross section is four times as large as $y_{b}^{2}$ in the $\geq 1 b$-jet scenario, to be compared to the factor of two in the inclusive case. Tighter $b$-jet requirements or the $p_{H}^{T}$ requirements only have the effect of further increasing the relative size of the $y_{t}^{2}$ contributions.

- It is interesting to notice that the $\geq 1 b b$ jet category, which requires one jet containing two bottom quarks, receives contributions essentially only from $y_{t}^{2}$ terms. This can be understood easily: a major part of the events for the loop-induced gluon fusion component features the Higgs recoiling against a hard gluon, which splits into a $b \bar{b}$ pair. The two bottom quarks in these configurations are boosted and generally close together, which makes it more likely for them to end up inside the same jet.

- Two opposed effects render the measurement of the bottom-quark Yukawa coupling in $b \bar{b} H$ production complicated: as pointed before the relative size of terms proportional to $y_{b}^{2}$ decreases as soon as $b$ jets are tagged. Nonetheless, tagging at least one of the $b$ jets is essential to distinguish $b \bar{b} H$ production from inclusive Higgs production, which predominantly proceeds via gluon fusion. ${ }^{7}$ Therefore, it is necessary to select suitable phase-space requirements which increase the relative $y_{b}^{2}$ contribution even in presence of at least one $b$ jet without loosing too much statistics. Given our findings for the $\geq 1 b b$-jet category, one can require at least one $b$ jet and veto all $b b$ jets. This decreases the $\geq 1 b$-jet rate for $y_{t}^{2}$ by roughly $20 \%$, while having a negligible effect on the $y_{b}^{2}$ rate. Below, we study differential distributions in order to find further requirements to enhance the relative size of the $y_{b}^{2}$ contributions.

- The LO contribution of the mixed $y_{b} y_{t}$ terms is negative in all scenarios, as has been observed already in ref. [30]. At NLO it yields a positive contribution only to

\footnotetext{
${ }^{7}$ Inclusive Higgs production may be used to extract $y_{b}$ only through the measurement of the inclusive Higgs transverse-momentum spectrum at very small $p_{H}^{T}$, see ref. [81] for example.
} 
the $\geq 1 b b$-jet scenario. The NLO/LO $K$-factor of the $y_{b} y_{t}$ term strongly depends on the scenario under consideration, which is expected given its interference-type contribution. By and large the impact of the $y_{b} y_{t}$ terms is minor though, reaching at most a few percent at NLO.

- Overall, QCD corrections have an even larger impact on the $y_{t}^{2}$ terms than on the $y_{b}^{2}$ terms, but they are quite sizable in either case. This can be understood as follows: as pointed out in ref. [30] potentially large logarithmic terms of $\log \left(m_{b}^{2} / m_{H}^{2}\right)$ enter the perturbative expansion of $y_{b}^{2}$ contributions in the $4 \mathrm{FS}$ and cause large perturbative corrections. Contributions proportional to $y_{t}^{2}$, on the other hand, feature a logarithmic enhancement of $g \rightarrow b \bar{b}$ splittings. These logarithms are taken into account for the first time up to NLO QCD in this paper, and yield an important correction to the cross section.

- Given the large QCD corrections, it is not surprising that perturbative uncertainties estimated from scale variations are relatively large as well. As expected they are largest for $y_{t}^{2}$ terms. The inclusion of NLO corrections reduces the uncertainties significantly, but they are still at the level $30 \%$ to $40 \%$. Their main source is again the logarithmic enhancement of the individual contributions pointed out above.

We now turn to discussing differential distributions. We first consider the NLO/LO $K$-factor of the different contributions to the cross section in figures 5 and 6 . These figures are organised according to the following pattern: there is a main frame, which shows histograms of the LO $y_{t}^{2}$ (green dashed), NLO $y_{t}^{2}$ (blue solid), LO $y_{b}^{2}$ (purple dash-dotted), and NLO $y_{b}^{2}$ (red dotted) predictions as cross section per bin (namely, the sum of the values of the bins is equal to the total cross section, possibly within cuts). In an inset we display the $K$-factor for each contribution by taking the bin-by-bin ratio of the NLO histogram which appears in the main frame over the LO one. The bands correspond to the residual uncertainties estimated from scale variations according to section 3.1.

Figure 5a shows the transverse momentum distribution of the Higgs boson. As expected from the general hardness of the two different production processes leading to $y_{t}^{2}$ and $y_{b}^{2}$ (the Higgs being radiated off a top-quark loop, and the Higgs boson being coupled to a bottom-quark line), the latter features the significantly softer $p_{H}^{T}$ spectrum. The $K$ factors for both contributions grow with the value of $p_{H}^{T}$ similarly, and become quite flat at large transverse momenta. However, as observed before, the size of QCD corrections is larger for the $y_{t}^{2}$ terms, ranging from $K \approx 2$ at small $p_{H}^{T}$ to $K \approx 3$ for $p_{H}^{T} \gtrsim 150 \mathrm{GeV}$. For $y_{b}^{2}$ contributions they are $K \approx 1.5$ and $K \approx 2$ in the same regions.

Also the transverse-momentum distribution of the leading $b$ jet in figure $5 \mathrm{~b}$ displays a harder spectrum for the $y_{t}^{2}$ contributions. Note that for the $p_{b_{1}}^{T}$ distribution, as we select the leading $b$ jet, the integral of the distribution corresponds to the $\geq 1 b$-jet rate. The behaviour of the $K$-factor is quite different in this case. While it is essentially flat and about $K=1.5$ for the $y_{b}^{2}$ terms, it is about $K=2.5$ for $y_{t}^{2}$ at low $p_{b_{1}}^{T}$, decreases to $K \approx 2$ for $p_{b_{1}}^{T}=150 \mathrm{GeV}$, and turns flat afterwards. 


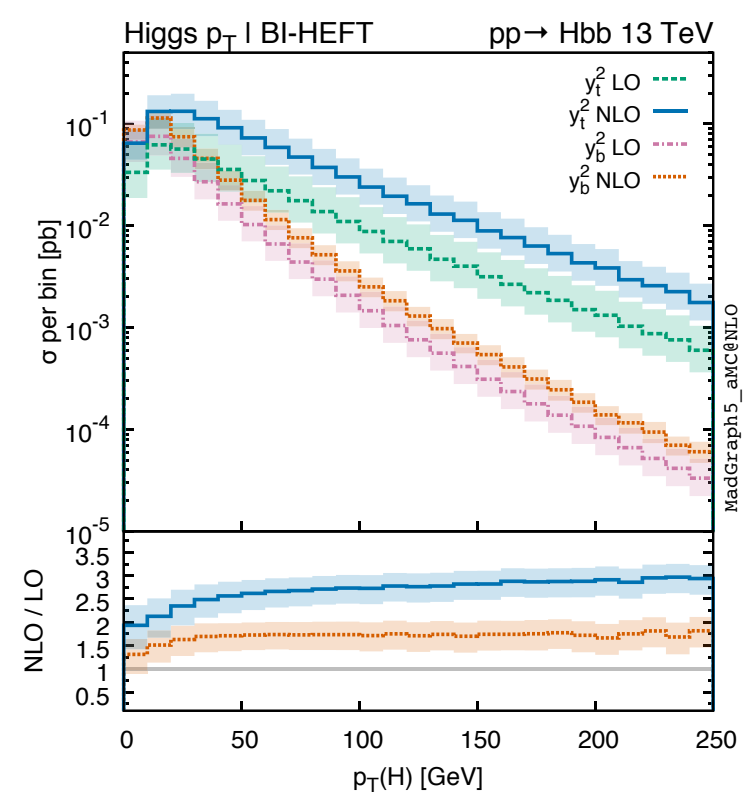

(a)

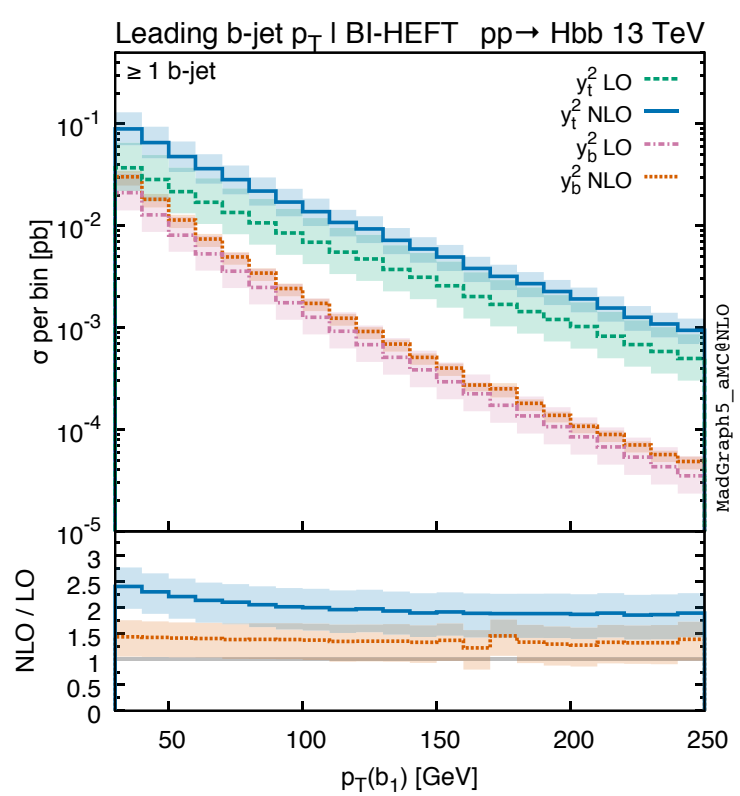

(b)

Figure 5. Distributions in the transverse momentum of the Higgs boson (5a) and of the hardest $b$ jet $(5 b)$. See the text for details.

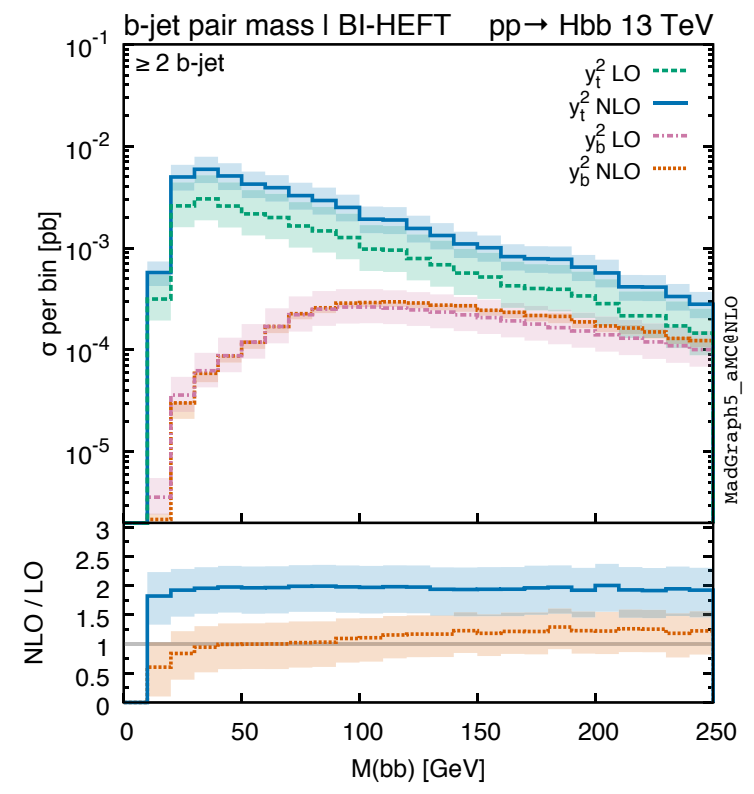

(a)

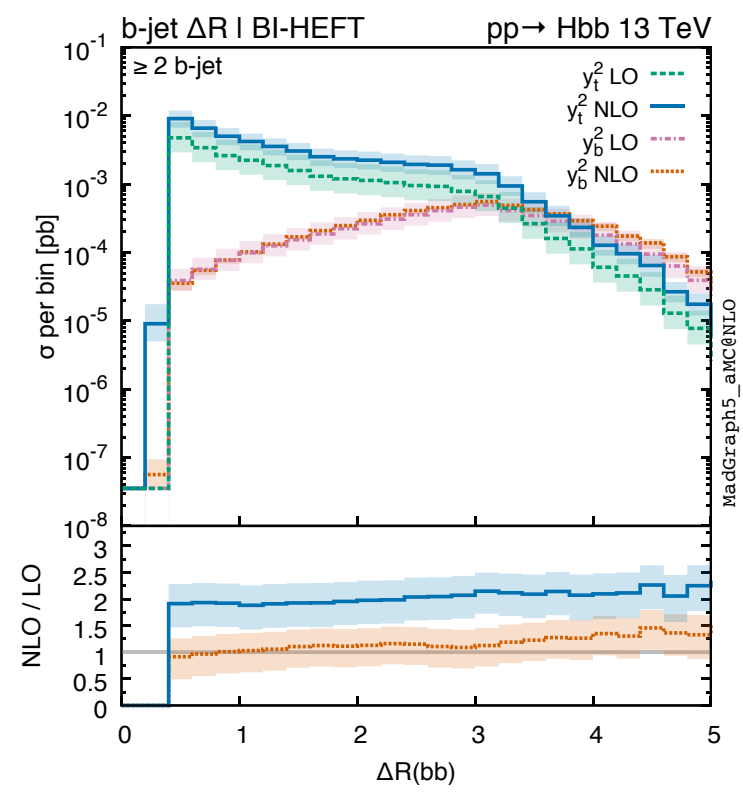

(b)

Figure 6. Same as in figure 5 , for the invariant mass of the two $b$ jets (6a) and their distance in the $\eta-\phi$ plane (6b). 
In figure 6 we consider two observables which require the presence of at least two $b$ jets: the left panel, figure $6 \mathrm{a}$, shows the invariant-mass distribution of the $b$-jet pair, $M(b b)$, and the right panel, figure $6 \mathrm{~b}$, shows their distance in the $\eta-\phi$ plane, $\Delta R(b b)$. It is interesting to notice the very different behaviour of $y_{b}^{2}$ and $y_{t}^{2}$ terms in the main frame of these two distributions. While $y_{t}^{2}$ clearly prefers small invariant-masses and small separations between the $b$ jets, $y_{b}^{2}$ peaks around $M(b b)=100 \mathrm{GeV}$ and $\Delta R(b b)=3$. The reason is clear: the dominant contribution for $y_{t}^{2}$ originates from the $g \rightarrow b \bar{b}$ splittings, which generate bottom-quark pairs that are hardly separated and, hence, also have a rather small invariant mass. Looking at the $K$-factors in the lower inset, the one of the $y_{b}^{2}$ terms turns out to be rather close to one for a large part of the phase space. It slightly increases with both $M(b b)$ and $\Delta R(b b)$. For $y_{t}^{2}$, on the other hand, the $K$-factor is around $K=2$, and shows an even milder increase with $M(b b)$ and $\Delta R(b b)$.

The scale-uncertainty bands in all four plots show the same features as the scale uncertainties discussed in table 1: their size decreases upon inclusion of higher-order corrections, but overall they are rather large even at NLO. The $y_{t}^{2}$ contributions feature a stronger scale dependence due to the logarithmic enhancement of $g \rightarrow b \bar{b}$ splittings. By and large, LO and NLO at least have some overlap in most cases. Nevertheless, $y_{b}^{2}$ contributions show the better converging perturbative series in that respect, which of course is directly related to their smaller QCD corrections.

\subsection{Accessing the bottom-quark Yukawa coupling}

We now return to the question of how to improve the sensitivity to the bottom-quark Yukawa coupling in $b \bar{b} H$ production. The goal is to increase the relative contribution of the $y_{b}^{2}$ terms by suitable selections, while keeping the absolute value of the cross section as large as possible. First, in order to be able to distinguish $b \bar{b} H$ from inclusive Higgs production, we require to observe at least one $b$ jet. Second, we have already noticed that by removing all $b$ jets containing a pair of bottom quarks, we can decrease the $y_{t}^{2}$ rate to some extent, with a negligible impact on the $y_{b}^{2}$ rate. The combination with additional phase-space requirements provides the most promising approach to further improve the sensitivity to the bottom-quark Yukawa coupling and to recover $b \bar{b} H$ production as the best process to measure $y_{b}$ directly. To this end, we consider the relative contribution of $y_{b}^{2}$ and $y_{t}^{2}$ terms to the $b \bar{b} H$ cross section for various differential observables in figures $7-9$. All the plots in these figures have a similar layout: the main frame shows NLO predictions for the $y_{t}^{2}$ contribution (blue dash-dotted), the $y_{b}^{2}$ contribution (red dotted), and the sum of all contributions, including the interference (black solid). The ratio inset shows the relative contributions of the $y_{b}^{2}$ and $y_{t}^{2}$ terms to $b \bar{b} H$ cross section. The bands reflect the residual uncertainties estimated from scale variations according to section 3.1.

We start in figure 7 with the transverse-momentum distribution of the Higgs boson. The three panels show this distribution with different requirements: figure 7 a displays the inclusive spectrum, figure $7 \mathrm{~b}$ is in the $\geq 1 b$-jet category, and figure $7 \mathrm{c}$ is in the same category, but vetoing $b b$ jets. This observable constitutes one of the strongest discriminators between $y_{b}^{2}$ and $y_{t}^{2}$ terms. The reason is the significantly softer spectrum of the terms proportional to $y_{b}^{2}$, which we already observed before. By looking at the three plots in 


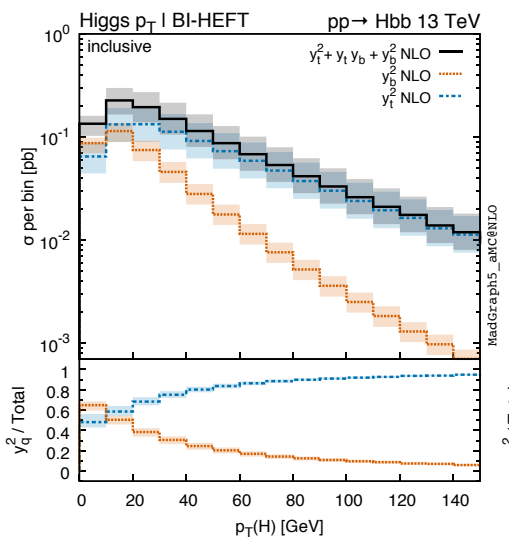

(a)

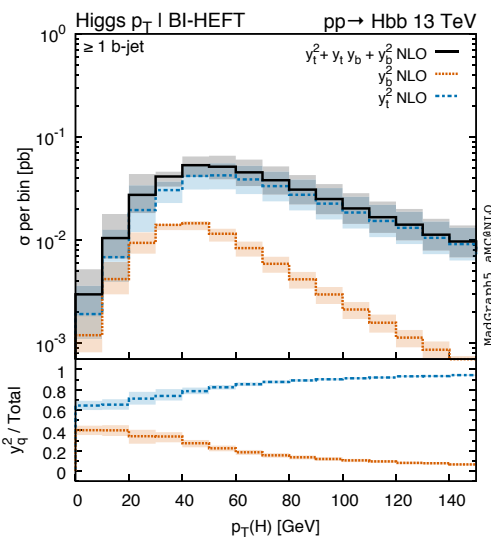

(b)

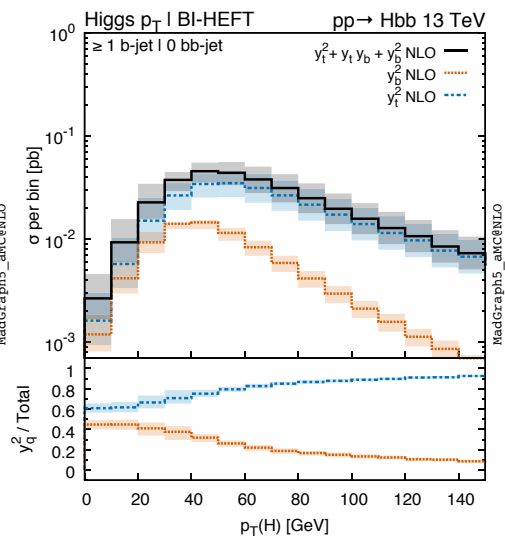

(c)

Figure 7. Distributions in the transverse momentum of the Higgs boson in three categories: inclusive $(7 \mathrm{a}), \geq 1 b$-jet $(7 \mathrm{~b})$, and $\geq 1 b$-jet $\mid 0 b b$-jets (7c). See the text for details.

figure 7 one can infer that the relative $y_{b}^{2}$ contribution is maximal in the inclusive case and at low Higgs transverse momentum, even exceeding $50 \%$ in the lowest part of the spectrum. If we require at least one $b$ jet the situation becomes worse, with the $y_{b}^{2}$ term reaching at most $40 \%$, again in the lowest transverse-momentum bins. If we require at least one $b$ jet and veto $b b$ jets, the relative contribution of $y_{b}^{2}$ at low transverse momentum is mildly increased. In the three cases (inclusive, $\geq 1 b$ jet and $\geq 1 b$ jet without $b b$ jets) the relative $y_{b}^{2}$ contribution quickly decreases with $p_{H}^{T}$, being less than $20 \%$ already at $p_{H}^{T}=50 \mathrm{GeV}$. At the level of the cross section, in the $\geq 1 b$-jet category, the relative $y_{t}^{2}$ and $y_{b}^{2}$ contributions are respectively $81 \%$ and $19 \%$. In the $\geq 1 b$-jet and no $b b$-jet category their relative contributions become $\sim 77 \%$ and $\sim 23 \%$, respectively. All in all, the gain coming from vetoing $b b$ jets is moderate. Another strategy, which can be combined with the $b b$-jet veto, consists in discarding events with the Higgs transverse momentum larger than a given value. For example, with an upper cut on $p_{H}^{T}$ at $50(100) \mathrm{GeV}$, in the category with at least one $b$ jet and no $b b$ jet, we can increase the relative contribution of $y_{b}^{2}$ terms to about $36 \%(27 \%)$, while keeping about $50 \%(90 \%)$ of its rate. Hence, restricting the phase space to small $p_{H}^{T}$ values allows us to increase the relative size of $y_{b}^{2}$ terms, while the impact on the rate is moderate due to the quite strong suppression at large $p_{H}^{T}$.

We continue in figure 8 with the transverse-momentum distribution of hardest $b$ jet. The general features of the $p_{b_{1}}^{T}$ spectrum are similar to the ones of $p_{H}^{T}$. However, as this observable clearly does not help very much in distinguishing between $y_{b}^{2}$ and $y_{t}^{2}$ contributions, we do not suggest any additional cut on $p_{b_{1}}^{T}$. It becomes clear from these plots, though, that a lower $p_{b}^{T}$ threshold used in the definition of $b$ jets would increase the relative size of the $y_{b}^{2}$ terms. In the present study jets are defined with a $p_{j}^{T}$ threshold of $30 \mathrm{GeV}$. A value of $25 \mathrm{GeV}$ or even $20 \mathrm{GeV}$ could be feasible at the LHC, and would further increase the sensitivity to the bottom-quark Yukawa coupling in $b \bar{b} H$ production. We note that additional modifications of the $b$-jet definition, for example the usage of a different jet radius 


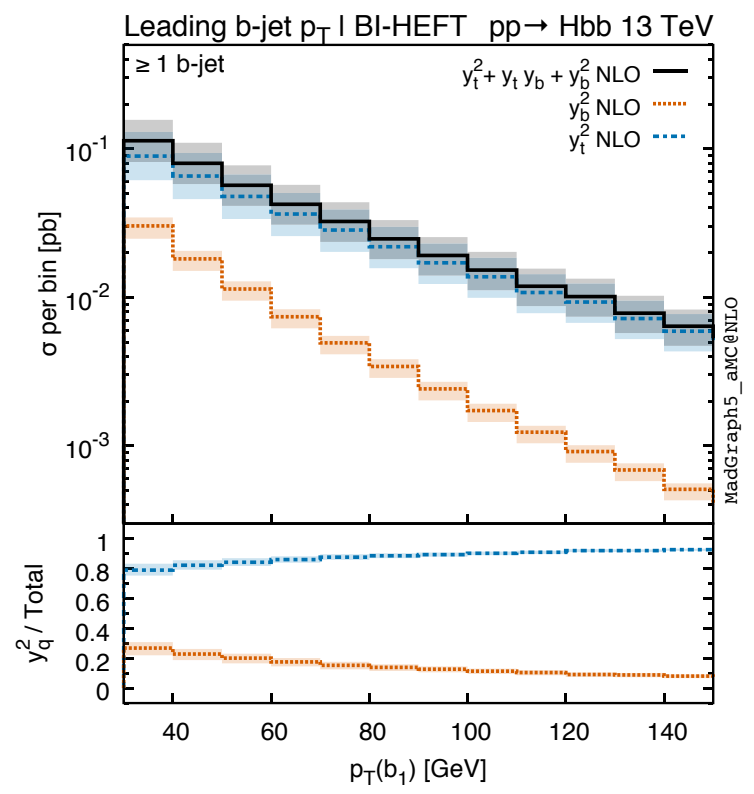

(a)

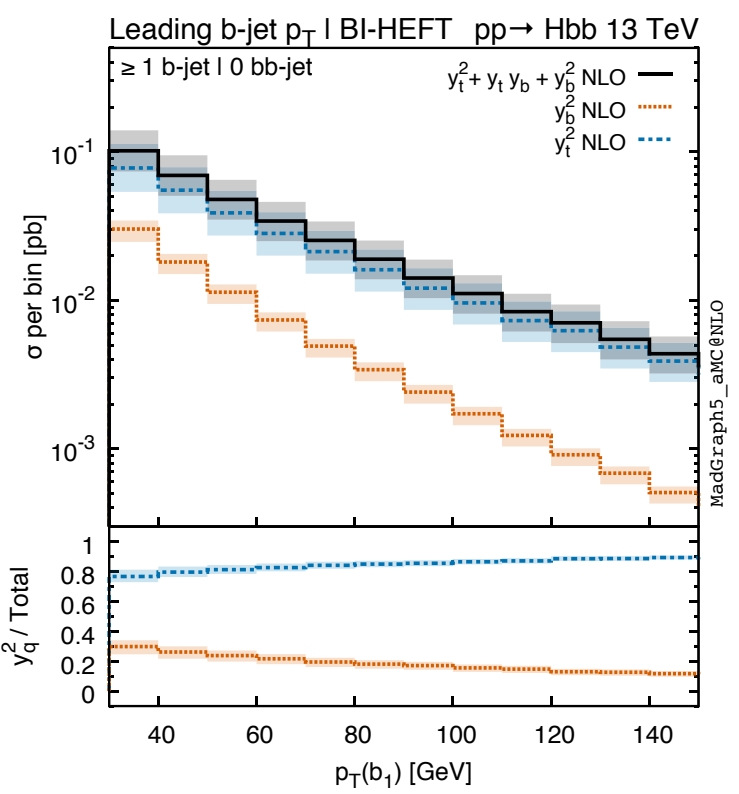

(b)

Figure 8. Same as figure 7, for the transverse momentum of the hardest $b$ jet. Note that the inclusive and $\geq 1$ b-jet categories yield identical distributions and we show only the latter.

(as shown in appendix A), or of jet-substructure techniques, can provide further handles to improve the discrimination of the $y_{b}^{2}$ contribution.

Finally, we consider figure 9 , where we show the invariant-mass distributions of the two $b$ jets, figure $9 \mathrm{a}$, and their distance $R$, figure $9 \mathrm{~b}$, and the corresponding distributions for $B$ hadrons, figures $9 \mathrm{c}$ and $9 \mathrm{~d}$. Note that the $M(b b)$ and $\Delta R(b b)$ distributions by construction require the presence of two $b$ jets, while $M(B B)$ and $\Delta R(B B)$ are shown with at least one $b$ jet and no $b b$ jet. Clearly, all of these observables, especially those related to $B$ hadrons, could in principle provide information to discriminate between $y_{b}^{2}$ and $y_{t}^{2}$ contributions. However, in practice, their usefulness is limited, due to two main reasons, both related to statistics. First, the two $b$-jet distributions require the presence of at least two $b$ jets and the corresponding rate is significantly reduced (by roughly one order of magnitude) with respect to the $\geq 1 b$-jet one. Second, the bulk of the $B$-hadron distributions feature $B$ hadrons which are quite soft, and therefore possibly not accessible in the measurements. We therefore conclude that, despite showing useful features, neither of these distributions can significantly help in obtaining additional sensitivity to the bottom-quark Yukawa coupling.

\subsection{QCD corrections to $g \rightarrow b \bar{b}$ splitting}

Besides its phenomenological relevance for the extraction of the bottom-quark Yukawa or as a background to other Higgs production processes at the LHC, $b \bar{b} H$ production induced by the top-quark Yukawa coupling offers a clean and simple theoretical setting to study the dynamics of $g \rightarrow b \bar{b}$ splitting in presence of a hard scale. Cases of interest at the LHC where such splitting plays an important role, and is in fact one of the main sources 


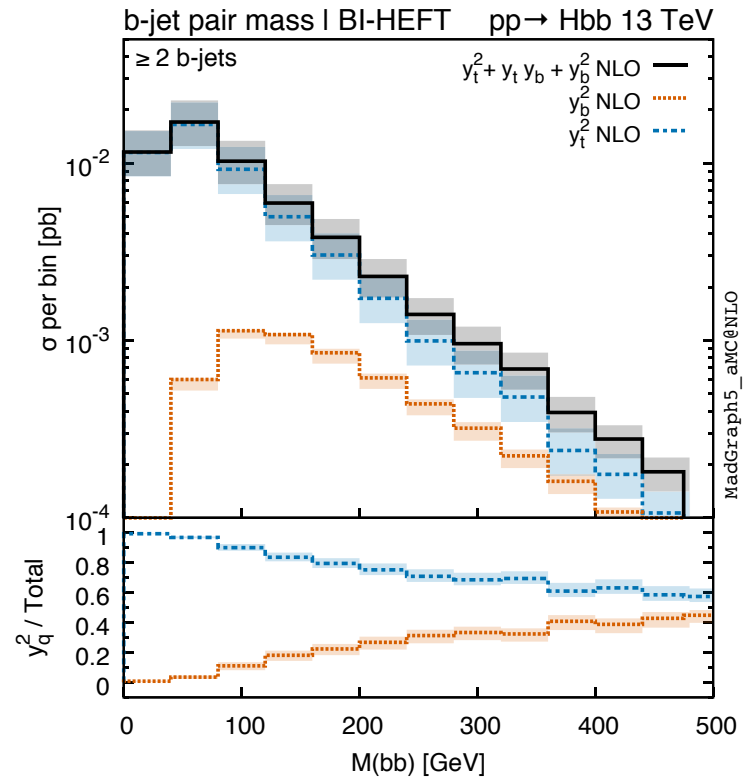

(a)

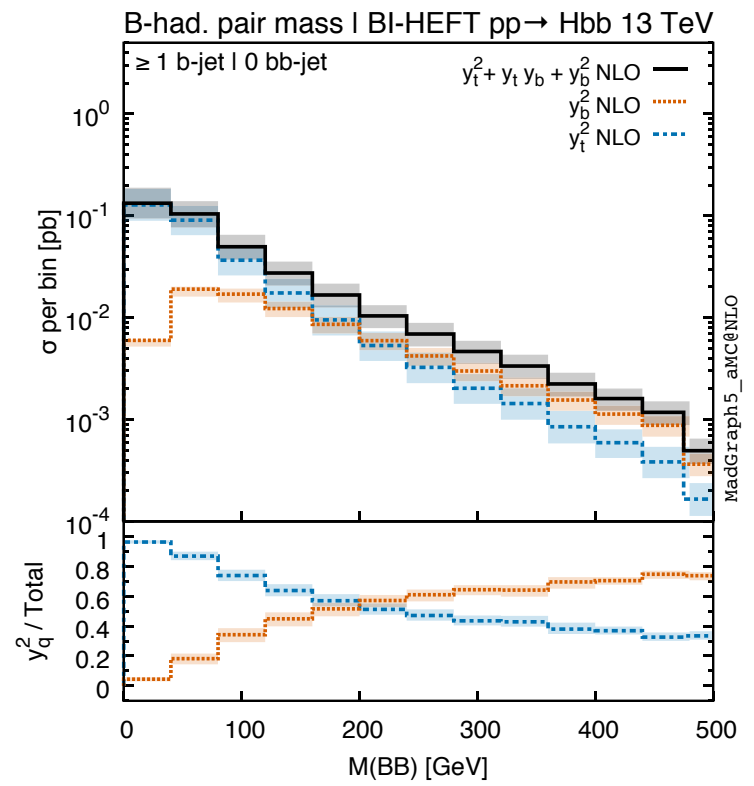

(c)

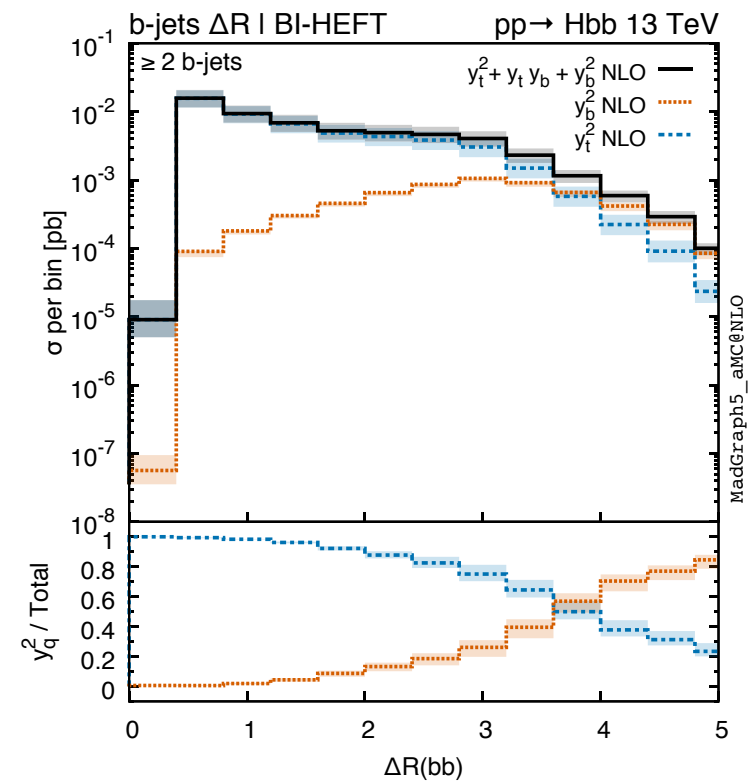

(b)

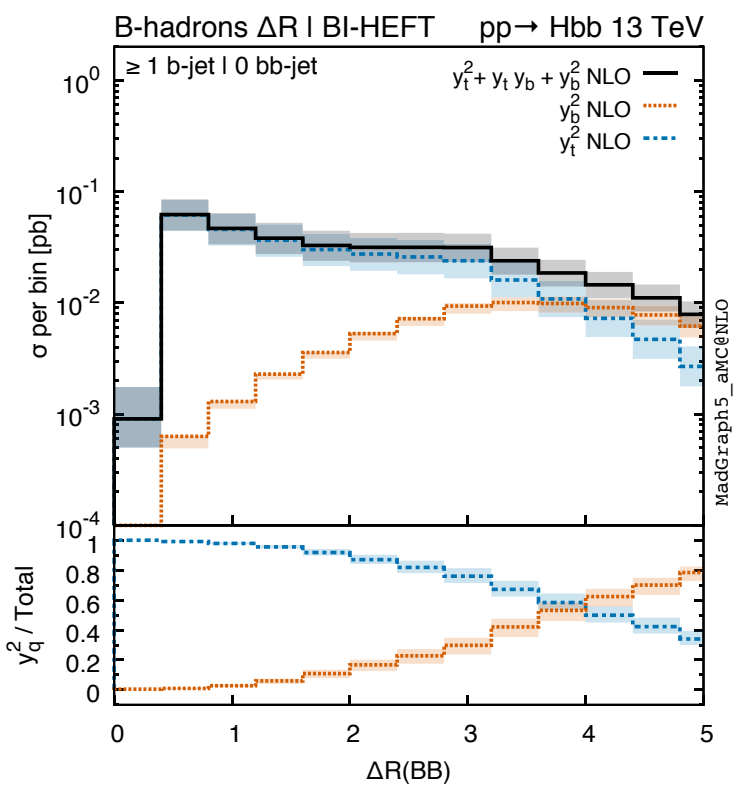

(d)

Figure 9. Same as figure $7 \mathrm{c}$, for the invariant mass of the two $b$ jets (9a), their distance in the $\eta-\phi$ plane (9a), and the same distributions for $B$ hadrons (9c and $9 d)$. 
of uncertainties, are $t \bar{t} b \bar{b}[82,83]$ and $b \bar{b} Z$ [49, 84], i.e., irreducible backgrounds for Higgs production in the $t \bar{t} H$ and $Z H$ production modes, respectively. In these cases, however, the production of a pair of $b$ quarks proceeds through different mechanisms and it is difficult to study them independently. ${ }^{8}$

In the previous sections, we have found that $b \bar{b} H$ production is dominated by the topquark Yukawa contribution. At the lowest order of the $y_{t}^{2}$ contribution the Higgs boson recoils against the $b \bar{b}$ pair coming from a gluon splitting, either in the initial or in the final state. When the splitting occurs in the initial state, a gluon typically produces a $b$ quark going forward and the other interacting at high $Q^{2}$, while when the splitting happens in the final state, a gluon has already been scattered at high $Q^{2}$. Therefore, asking a pair of $b$ quarks at high $p^{T}$ mostly selects the mechanism of gluon splitting in the final state.

This LO picture is modified at NLO, in particular in presence of real radiation where additional configurations can appear: most importantly, the Higgs can recoil against a hard light parton, with the $b \bar{b}$ pair being soft/collinear. Such configurations can give a large contribution as they are possibly enhanced by soft and/or collinear logarithms. In fact, rather than treating them as NLO corrections to $b \bar{b} H$, such contributions can be thought as higher-order corrections to Higgs + jet production. In this case, they can be described either with a parton-shower or by employing a gluon fragmentation function and its evolution. Both approaches resum (with different accuracy) large logarithms of the form $\log \left(p^{T} / m_{b}\right)$, with $p^{T}$ being the transverse momentum of the light parton.

The $y_{t}^{2}$ contribution to $b \bar{b} H$ production enables a direct assessment of the importance of these configurations. This can be done by studying very simple observables. To this aim, we consider the fraction of energy (or equivalently of transverse momentum) of the $b$ jet which is carried by particles other than the $b$ quark. At NLO accuracy, where only one extra light parton, dubbed $g$, can be emitted, this fraction can be defined for the $i$-th $(b)$ jet as

$$
z_{g}\left(j_{i}\right)=\frac{p_{g}^{T}}{p_{j_{i}}^{T}} .
$$

Jets featuring hard $b$ quarks and soft gluon emissions are characterised by $z_{g} \ll 1$, while $b$ jets with a hard light parton and soft $b$ quarks yield $z_{g} \simeq 1$. In the latter case, one would rather consider the $b$ quark to be originated from the evolution of the light parton. The limiting cases are easily identified: $z_{g}=0$ means that the jets are constituted only by one or two b quarks, while $z_{g}=1$ corresponds to light jets where $g$ is the only constituent.

In the following, we consider the momentum fraction carried by light partons in the hardest $b$ jet, $z_{g}\left(b_{1}\right)$. We start by showing, in figure 10 , the (normalised) $z_{g}\left(b_{1}\right)$ distribution for the $y_{b}^{2}$ (red dotted) and $y_{t}^{2}$ (blue dash-dotted) contributions to $b \bar{b} H$ production, as well as the complete $b \bar{b} H$ cross section (black solid). The different behaviour in the two cases is manifest: for the $y_{b}^{2}$ contribution, $z_{g}\left(b_{1}\right)$ is monotonically decreasing, and configurations with a hard gluon inside the $b$ jet are very suppressed. On the contrary, the $y_{t}^{2}$ contribution shows a plateau in the range $0.6<z_{g}\left(b_{1}\right)<0.95$. The integrated cross section for $z_{g}\left(b_{1}\right)>$ 0.6 amounts to $1.8 \%$ of the $\geq 1 \mathrm{~b}$-jet one.

\footnotetext{
${ }^{8}$ One must bear in mind that besides applications in certain LHC processes, proper modelling of $g \rightarrow b \bar{b}$ splitting plays an important role also in the context of parton showers.
} 


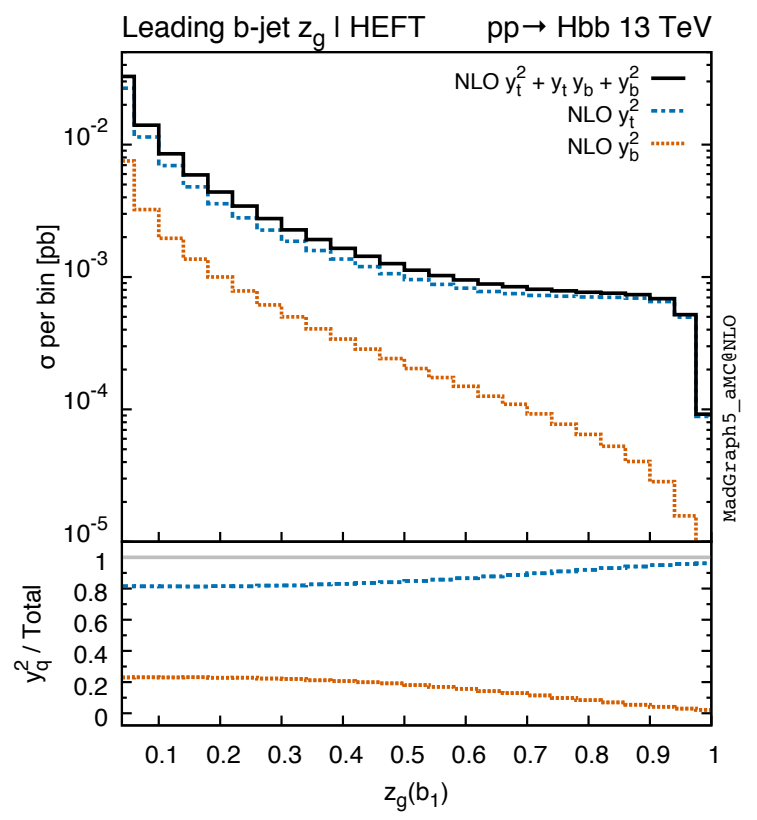

Figure 10. The momentum fraction of the first $b$ jet carried by light partons, $z_{g}^{b_{1}}$ in $b \bar{b} H$ production.

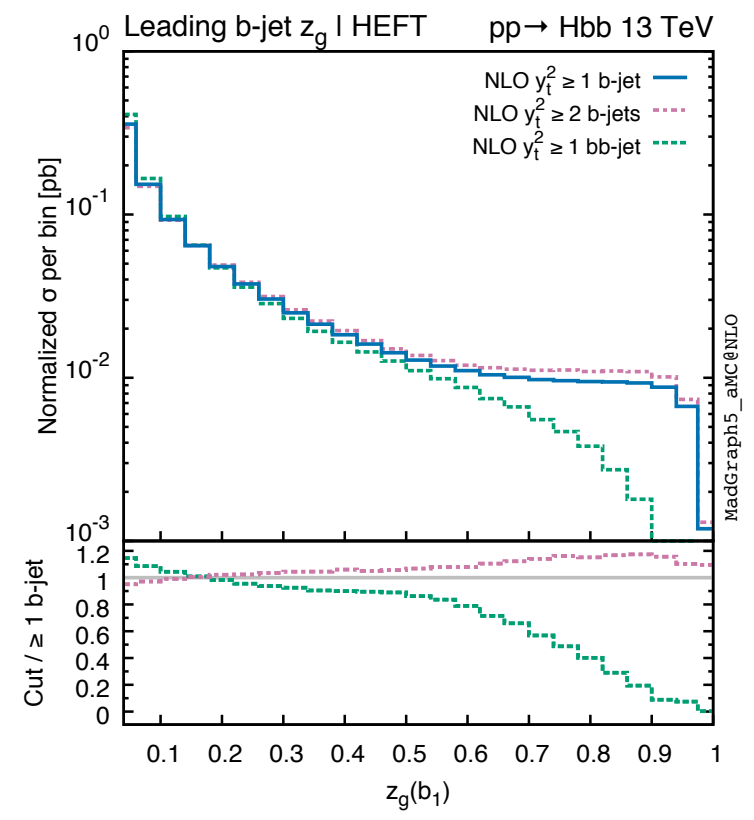

(a)

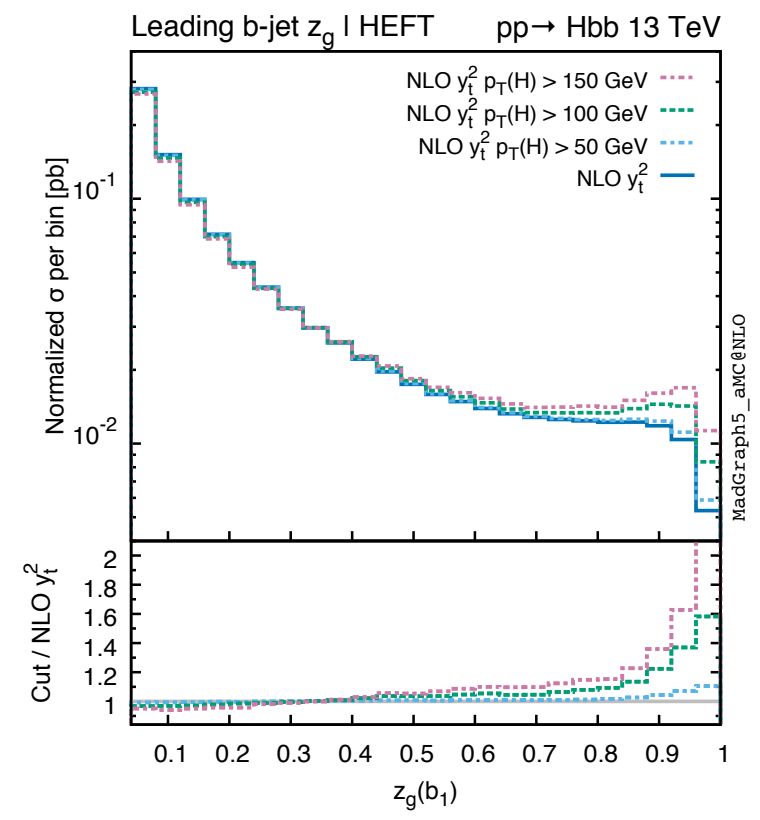

(b)

Figure 11. The momentum fraction of the first $b$ jet carried by light partons, $z_{g}^{b_{1}}$, for the $y_{t}^{2}$ contribution to $b \bar{b} H$ production, for different $b$-jet acceptances (11a) and different Higgs- $p^{T}$ cuts (11b).

Next, we focus on the $y_{t}^{2}$ contribution, and consider the $z_{g}\left(b_{1}\right)$ distribution with different acceptance cuts. In figure 11a, we show the jet acceptances considered in section 3.2 and two $b$ jets while in figure 11b the Higgs transverse-momentum cuts are shown. From the first figure, we conclude that requiring a $b b$ jet leads to a suppression of configurations with a hard light parton. This is reflected in the mild shape enhancement for $z_{g}\left(b_{1}\right) \simeq 1$ 
when a second, separate $b$ jet is required. In the second figure we appreciate how, at large Higgs transverse momenta, configurations with a hard light parton are more and more enhanced. When a minimum $p_{H}^{T}$ cut of $100 \mathrm{GeV}$ is required, $z_{g}\left(b_{1}\right)$ even starts to increase as $z_{g}\left(b_{1}\right)>0.8$. In this case, the fraction of cross section for $z_{g}\left(b_{1}\right)>0.6$ is $2.3 \%$, and it reaches $3 \%$ for $p_{H}^{T}>150 \mathrm{GeV}$. Although the relative importance of such configurations with respect of the total rate is marginal, the enhancement is manifest.

Being aware of the limitations of a fixed-order approach in this context, our findings could motivate a more complete study to explore the possibility of enhancing the $y_{b}^{2}$ contribution to $b \bar{b} H$ production over the $y_{t}^{2}$ one by requiring, for instance, an energy threshold for the $B$ hadron inside the hardest $b$ jet, and/or vetoing double b-tagged jets. Alternatively, substructure techniques could be employed to reveal the internal details of jets and classify events more efficiently.

\section{Summary and outlook}

The precise determination of the Higgs-boson couplings will be one of the main goals of the LHC programme of the coming decades. In this work we have presented for the first time the computation of the contributions proportional to the top-quark Yukawa coupling to $b \bar{b} H$ production at NLO in QCD using the $4 \mathrm{FS}$. Given that the exact NLO computation is beyond the reach of the current multi-loop technology, we have employed an effective field theory approach, where the top quark is integrated out and the Higgs boson couples directly to gluon fields via a dimension-five operator. In order to reach NLO accuracy in the HEFT, in addition to the usual tree-level, virtual and real terms, we have also determined the finite $O\left(y_{t}\right)$ corrections to the bottom-quark Yukawa, by matching the HEFT to the full theory at two loops. Our results agree with previous calculations available in literature [66-68]. We have argued that the HEFT approximation is suitable to describe the phase space region where the bulk of the $b \bar{b} H$ cross section resides, i.e., for the Higgs boson, up to $p_{H}^{T} \simeq 200 \mathrm{GeV}$.

The main result of our study is that the $b \bar{b} H$ final state is largely dominated by the top-quark Yukawa contributions in all regions of the phase space, at least where the Bornimproved HEFT approximation can be trusted. This is contrary to the common lore and intuition that the $b \bar{b} H$ final state gives direct access to $y_{b}^{2}$, as much as $t \bar{t} H$ gives access to $y_{t}^{2}$. The failure of such simple-minded approach is mostly due to the large hierarchy between $y_{t}^{2} / y_{b}^{2}$ which makes up for the relative $\alpha_{s}^{2}$ and loop-squared suppression of the top-induced contribution. While being expected to some extent by existing LO computations of the $y_{t}^{2}$ terms, such conclusion becomes glaring (and robust) at NLO due to the very large $K$-factor (almost 3) associated to the $y_{t}^{2}$ contribution. Apart from the decreased sensitivity to the bottom-quark Yukawa coupling, this result also entails a larger cross section of associated $b \bar{b} H$ production and henceforth a better chance of measuring $b \bar{b} H$ at the LHC.

We have then investigated in detail how the two main contributions, those proportional to $y_{b}^{2}$ and $y_{t}^{2}$, can be disentangled by using suitable observables and selection cuts. By systematically studying kinematical distributions of the final states at NLO accuracy, we have identified two main handles: first, the largest relative contribution from $y_{b}^{2}$ terms 
resides at small Higgs transverse momentum. Second, the $y_{t}^{2}$ terms are strongly dominated by configurations with gluon splitting into a $b \bar{b}$ pair, appearing already at LO, often leading to a high- $p^{T}$ jet consisting of two bottom quarks. Our results indicate that the $y_{t}^{2}$ contribution will always provide a significant fraction of the irreducible background to the $y_{b}^{2}$ one, and therefore the $y_{t}^{2}$ contribution should be included in global fits, such as those based on the rescaling of SM couplings (kappa framework) [85].

Finally, predictions for $b \bar{b} H$ total and differential rates at NLO in QCD have a wide range of phenomenological relevance and applications, which go beyond what was discussed in this work and are worth exploring in the future. The first natural extension of our work will be to promote the NLO results to fully exclusive ones by matching to parton showers. While technically straightforward, this step entails understanding and controlling the interplay between fixed-order and resummed radiation from the bottom quarks as well as the gluon-splitting mechanism, a topic which has been the subject of several recent investigations $[82-84,86]$. As briefly explored in this work, the $y_{t}^{2}$ contribution is very sensitive to $g \rightarrow b \bar{b}$ splittings and therefore could provide an optimal testing bench for further studies. The second extension will be a careful reassessment of $b \bar{b} H$ as potential background to other final states featuring the Higgs boson, in the SM measurements as well as in Beyond the SM (BSM) searches, mostly due to the large enhancement of the cross section at NLO. For instance, in the search for $t \bar{t} H$ with $H \rightarrow \gamma \gamma$, only very weak constraints on additional ( $b$-)jet activity beyond the two photons (on the Higgs mass shell) are required, and $b \bar{b} H$ production can contribute to the signal region. Similarly, $H H$ searches where at least one of the Higgs bosons decays into a $b \bar{b}$ pair will be affected by associated $b \bar{b} H$ production, as the $b \bar{b} H$ rate with $m_{b b}>100 \mathrm{GeV}$ is comparable to the $H H$ cross section and it is dominated by $y_{t}^{2}$ terms as soon as $H$ has a moderate $p^{T}$. Other channels, currently searched for in $3 b$-jet final states, such as associated heavy-quark and double-scalar production in extensions of the SM like the 2HDM, could also be affected by a sizable $b \bar{b} H$ background.

\section{Acknowledgments}

We are grateful to Sasha Nikitenko and Nikos Romptis for discussions and information on the details of the measurements. MW thanks Michael Spira for many useful insights and discussions on Higgs production in association with bottom quarks. ND is thankful to Claude Duhr for his essential advice and guidance and many discussions on how to perform the two loop calculation reported in this work and to him and Falko Dulat for providing a version of POLYLOGTOOLs. The work of ND has been supported by the ERC grants 637019 'MathAm' and 694712 'pertQCD'. ND further acknowledges the hospitality of the CERN TH department while this work was carried out. FM has received fundings from the European Union's Horizon 2020 research and innovation programme as part of the Marie Skłodowska-Curie Innovative Training Network MCnetITN3 (grant agreement no. 722104) and by the F.R.S.-FNRS under the 'Excellence of Science' EOS be.h project n. 30820817. The work of MW is supported by the ERC Consolidator Grant 614577 HICCUP. The work of MZ is supported by the Netherlands National Organisation for Scientific Research (NWO). 


\begin{tabular}{|c|c|c|c|c|}
\hline & $R=0.4$ & LO (acceptance) & NLO (acceptance) & $K$ \\
\hline \multirow{2}{*}{$\geq 1 b$} & $y_{t \mathrm{HEFT}}^{2}$ & $1.70 \cdot 10^{-1}{ }_{-39 \%}^{+72 \%}(47 \%)$ & $3.67 \cdot 10^{-1}{ }_{-29 \%}^{+39 \%}(42 \%)$ & 2.2 \\
\hline & $y_{b}^{2}$ & $6.02 \cdot 10^{-2}{ }_{-31 \%}^{+52 \%}(23 \%)$ & $8.49 \cdot 10^{-2}{ }_{-16 \%}^{+13 \%}(21 \%)$ & 1.4 \\
\hline \multirow{2}{*}{$\geq 2 b$} & $y_{t \text { HEFT }}^{2}$ & $2.48 \cdot 10_{-39 \%}^{-2}+72 \%(6.9 \%)$ & $4.86 \cdot 10^{-2}{ }_{-27 \%}^{+33 \%}(5.5 \%)$ & 2.0 \\
\hline & $y_{b}^{2}$ & $5.07 \cdot 10_{-31 \%}^{-3}+51 \%(1.9 \%)$ & $\left.5.92 \cdot 10_{-12 \%}^{-3}+1.5 \%\right)$ & 1.2 \\
\hline \multirow{2}{*}{$\geq 1 b b$} & $y_{t}^{2} \mathrm{HEFT}$ & $\left.3.84 \cdot 10_{-38 \%}^{-2}+711 \%\right)$ & $7.86 \cdot 10^{-2}{ }_{-28 \%}^{+36 \%}(8.9 \%)$ & 2.0 \\
\hline & $y_{b}^{2}$ & $3.37 \cdot 10^{-4}{ }_{-34 \%}^{+57 \%}(0.1 \%)$ & $2.53 \cdot 10^{-4}{ }_{-48 \%}^{+4 \%}(0.1 \%)$ & 0.7 \\
\hline
\end{tabular}

\begin{tabular}{|c|c|c|c|c|}
\hline & $R=1$ & LO (acceptance) & NLO (acceptance) & $K$ \\
\hline \multirow{2}{*}{$\geq 1 b$} & $y_{t \text { HEFT }}^{2}$ & $1.80 \cdot 10^{-1}{ }_{-39 \%}^{+72 \%}(50 \%)$ & $4.16 \cdot 10^{-1}{ }_{-30 \%}^{+43 \%}(47 \%)$ & 2.3 \\
\hline & $y_{b}^{2}$ & $6.08 \cdot 10^{-2}{ }_{-31 \%}^{+52 \%}(23 \%)$ & $\left.9.23 \cdot 10_{-18 \%}^{-2}+23 \%\right)$ & 1.5 \\
\hline \multirow{2}{*}{$\geq 2 b$} & $y_{t}^{2}$ HEFT & $\left.1.46 \cdot 10_{-39 \%}^{-2}+4.13 \%\right)$ & $3.32 \cdot 10^{-2}{ }_{-30 \%}^{+42 \%}(3.8 \%)$ & 2.3 \\
\hline & $y_{b}^{2}$ & $\left.4.89 \cdot 10_{-31 \%}^{-3}+1.91 \%\right)$ & $\left.6.80 \cdot 10_{-16 \%}^{-3}+1.7 \%\right)$ & 1.4 \\
\hline \multirow{2}{*}{$\geq 1 b b$} & $y_{t \mathrm{HEFT}}^{2}$ & $8.73 \cdot 10^{-2}{ }_{-38 \%}^{+71 \%}(24 \%)$ & $1.91 \cdot 10_{-29 \%}^{-1}+39 \%(22 \%)$ & 2.2 \\
\hline & $y_{b}^{2}$ & $2.22 \cdot 10^{-3}{ }_{-34 \%}^{+57 \%}(0.8 \%)$ & $2.13 \cdot 10^{-3}{ }_{-19 \%}^{+1 \%}(0.5 \%)$ & 1.0 \\
\hline
\end{tabular}

Table 2. Cross sections (in $\mathrm{pb}$ ) for different $b$-jet multiplicities with jet radius $R=0.4$ (top table) and $R=1$ (bottom table). For $R=0.4$, numbers are the same as in table 1 .

\section{A Jet rates with $R=1$}

In this appendix we show results for the jet categories considered in section 3 using $R=1$ as jet-radius parameter. The choice of such a value is motivated by boosted-Higgs searches. More in general, a larger jet-radius enhances the $\geq 1 b b$-jet category, thus making a more efficient reduction of the $y_{t}^{2}$ contribution possible. Apart from the jet-radius parameter, we employ exactly the same setup as described in section 3.1.

Our results are shown in table 2. For convenience of the reader, we also quote in the upper part of the table the jet rates computed with $R=0.4$ (taking the results directly from table 1), while the bottom part of the table displays results with $R=1$. In order to keep the table minimal, we do not show the $y_{b} y_{t}$ interference and the BI-HEFT contribution, nor the sum of all contributions to the total cross section. As displayed in table 1, the effect of including the full top-mass dependence in the jet rates is small, in particular on the acceptance.

The conclusions that can be drawn from the table are the following:

- Choosing $R=1$ has the effect of mildly increasing the $\geq 1 b$-jet category, with a slightly more pronounced effect for the $y_{t}^{2}$ contribution than for the $y_{b}^{2}$ one. This can be easily understood as $R=1$ makes it possible to cluster the radiation more 
inclusively. Events with both $b$ quarks slightly below the jet- $p^{T}$ threshold may more easily lead to a $b$ jet with a larger jet radius. However, one should also keep in mind acceptance effects: if one of the $b$ quark is outside the jet-acceptance rapidity window, the resulting jet may not fall inside the acceptance, and hence be discarded. The fact that the cross section is larger with $R=1$ than with $R=0.4$ hints that acceptance effects should be less important than the inclusive clustering of the radiation. However, the former effects explain why the exclusive one- $b$ jet category (obtained by subtracting the $\geq 2 b$ and $\geq 1 b b$ from the $\geq 1 b$ one) is larger for $R=0.4$ than for $R=1$, as it can be trivially computed from the numbers in the table.

- Concerning the $\geq 2 b$-jet category, the larger jet radius leads to a relative $30-40 \%$ reduction of the $y_{t}^{2}$ rate. The $y_{b}^{2}$ term rate is slightly reduced at LO while it is increased of about $10 \%$ (relative) at NLO. Again, two competing effects should be considered in order to explain the behaviour: on the one hand, a larger jet radius requires the $b$ jets to be more separated, leading to a reduction of the $\geq 2 b$-jet rate; on the other, it leads to a more inclusive clustering of the QCD radiation, thus instead giving an increase of the rate. Given the tendency of the two $b$ jets to lie closer in the $y_{t}^{2}$ than in the $y_{b}^{2}$ contribution to the cross section (see also figure $6 \mathrm{~b}$ ), the first effect will be more pronounced on the former contribution, and the second on the latter.

- Finally, the $\geq 1 b b$ category is where the effect of using $R=1$ is most pronounced. The relative increase of the $y_{b}^{2}$ contribution is very large, more than a factor 6 (8) at LO (NLO). However, the absolute rate remains negligible to all practical purposes, with an acceptance below 1\%. For the $y_{t}^{2}$ contribution the relative increase is still large, about a factor 2 , and the acceptance now exceeds $20 \%$.

The above remarks support vetoing fat $b b$ jets as a way to further reduce the effect of the $y_{t}^{2}$ term and thus increase the sensitivity on the bottom-quark Yukawa. As we already mentioned in the conclusions of our work, the natural follow-up of these findings would be to perform a more detailed study based on fully exclusive final state, which includes matching with parton showers.

\section{B Matching and renormalization schemes}

The matching of the HEFT to the SM for the computation of higher-order corrections requires a well-defined renormalization scheme in both theories. In practice, rather than considering the full SM, one can focus only on the part relevant for the study of the $p p \rightarrow b \bar{b} H$ process, at the order under consideration. Therefore, as it is customary in the literature [66, 67], we can restrict ourselves to a simplified model, featuring QCD with four massless and two massive quarks and a singlet scalar $H$ that couples to the massive quarks. Hence we do not have to deal with issues related to the breaking of SU(2) gauge invariance or the relation between Yukawa couplings and masses. The Lagrangian of this 
model, which for brevity is denoted as SM, has the following form:

$$
\begin{aligned}
\mathcal{L}_{\mathrm{SM}}= & -\frac{1}{4} G_{\mu \nu} G^{\mu \nu}+\frac{1}{2}\left(\partial_{\mu} H \partial^{\mu} H-m_{H}^{2} H^{2}\right)-V(H) \\
& +\sum_{\psi=u, d, c, s} i \bar{\psi} \not D \psi+\sum_{\Psi=b, t}\left[i \bar{\Psi}\left(\not D-m_{\Psi}\right) \Psi-\frac{y_{\Psi}}{\sqrt{2}} \bar{\Psi} \Psi H\right]+\mathcal{L}_{\mathrm{gf}}
\end{aligned}
$$

where $G_{\mu \nu}$ is the gluon field strength tensor for the gluon field $G_{\mu} . H$ is the scalar Higgs field with mass $m_{H}$. The four light quarks $\psi \in\{u, d, c, s\}$ and the two massive quarks $\Psi \in\{b, t\}$ are labelled by the usual SM flavor symbols. Their masses and Yukawa couplings are denoted by $m_{b}, m_{t}$ and $y_{b}, y_{t}$. Finally $\mathcal{L}_{\text {gf }}$ is the gauge-fixing and ghost Lagrangian of the gauge interaction. We denote the QCD coupling constant in this theory as $\alpha_{s}$.

The EFT to which we match our theory in the heavy-top quark limit is expressed by the following Lagrangian:

$$
\begin{aligned}
\mathcal{L}_{\mathrm{HEFT}}= & -\frac{1}{4} \tilde{G}_{\mu \nu} \tilde{G}^{\mu \nu}+\frac{1}{2}\left(\partial_{\mu} \tilde{H} \partial^{\mu} \tilde{H}-\left(m_{H}^{\mathrm{HEFT}}\right)^{2} \tilde{H}^{2}\right)-V(\tilde{H}) \\
& +\sum_{\tilde{\psi}=\tilde{u}, \tilde{d}, \tilde{c}, \tilde{s}} i \overline{\tilde{\psi}} \not D \tilde{\psi}+\left[i \overline{\tilde{b}}\left(\not D-m_{b}^{\mathrm{HEFT}}\right) \tilde{b}-\frac{\left.y_{b}^{\mathrm{HEFT}} \tilde{\tilde{b}} \tilde{b} \tilde{H}\right]+\mathcal{L}_{\mathrm{gf}}}{\sqrt{2}}\right. \\
& -\frac{C_{1}}{4} H \tilde{G}_{\mu \nu} \tilde{G}^{\mu \nu}+\sum_{i=2}^{5} C_{i} O_{i},
\end{aligned}
$$

where the fields with a tilde denote the low-energy analogue of the SM fields. The HEFTlabelled parameters have a SM equivalent to which they are matched at leading power. We consider next-to-leading power interactions, mediated by dimension-five interactions in the last line of eq. (B.2), but show only the first of the five independent operators that were first listed in ref. [87] as the others are not relevant for this paper (in particular they do not mix with $O_{1}$ under renormalization). While not used explicitly in eq. (B.2), we refer to the strong coupling constant in this theory as $\alpha_{s}^{\mathrm{HEFT}}$. The HEFT is equivalent to the original Lagrangian in the heavy-top quark limit if one can express the renormalized parameters of the HEFT and its renormalized fields in terms of those of the original theory such that amplitudes in the two theories are equal up to terms supressed by inverse powers of the top mass.

In the case at hand it is established that a particularly suitable scheme is the socalled decoupling scheme [88], which is generally used in Madgraph5_aMC@NLO. ${ }^{9}$ In this scheme, fields and masses are renormalized on-shell, and couplings are renormalized in a mixed way, where the counterterms that cancels UV divergences from loops with light degrees of freedom are subtracted in the $\overline{\mathrm{MS}}$ scheme, while those involving heavy quarks are renormalized on-shell. As a consequence, the running of the strong coupling is the same as in QCD with four flavors and $\overline{\mathrm{MS}}$ renormalization. The matching condition for $\alpha_{s}$ and the gluon field are then trivial. In our calculation, we differ from the standard Madgraph5_aMC@NLO scheme in that we renormalize the bottom-quark Yukawa coupling in the $\overline{\mathrm{MS}}$ scheme. Finally, also for the gluon-Higgs operator coupling $\overline{\mathrm{MS}}$ renormalization is

\footnotetext{
${ }^{9}$ See appendix B of ref. [89] for an explicit discussion.
} 
used. Let us write the relevant renormalization equations for the processes considered in this paper. We label all bare quantities with a superscript $B$.

$$
\begin{aligned}
\alpha_{s}^{\mathrm{HEFT}, B} & =\mu^{2 \epsilon} S_{\epsilon}^{-1} Z_{\alpha} \alpha_{s}^{\mathrm{HEFT}}, \\
m_{b}^{\mathrm{HEFT}, B} & =Z_{m_{b}} m_{b}^{\mathrm{HEFT}}, \\
y_{b}^{\mathrm{HEFT}, B} & =\mu^{\epsilon} S_{\epsilon}^{-1 / 2} Z_{y_{b}} y_{b}^{\mathrm{HEFT}}, \\
C_{1}^{B} & =\mu^{\epsilon} S_{\epsilon}^{-1 / 2} Z_{C} C_{1},
\end{aligned}
$$

where $S_{\epsilon}=\exp \left(-\gamma_{E} \epsilon\right)(4 \pi)^{\epsilon}$ with $\gamma_{E}$ the Euler-Mascheroni constant and the renormalization constants take the following form:

$$
\begin{aligned}
Z_{\alpha} & =1-\frac{\alpha_{s}^{\mathrm{HEFT}}}{4 \pi} \frac{1}{\epsilon}\left(\beta_{0}^{(4)}-\frac{2}{3}\left(\frac{\mu}{m_{b}^{\mathrm{HEFT}}}\right)^{2 \epsilon}\right), \\
Z_{m_{b}} & =1-\frac{\alpha_{s}^{\mathrm{HEFT}}}{\pi}\left(\frac{1}{\epsilon}+\frac{4}{3}\right), \\
Z_{y_{b}} & =1-\frac{\alpha_{s}^{\mathrm{HEFT}}}{\pi} \frac{1}{\epsilon} \\
Z_{C} & =1-\frac{\alpha_{s}^{\mathrm{HEFT}}}{4 \pi} \frac{1}{\epsilon}\left(\beta_{0}^{(5)}\right),
\end{aligned}
$$

where $\beta_{0}^{(n f)}=11-2 / 3 n_{f}$. The renormalized parameters in the HEFT are then expressed in terms of renormalized parameters of the full theory. The result of the matching is the following:

$$
\begin{aligned}
C_{1} & =\frac{\alpha_{s}}{3 \pi}\left(\frac{y_{t}}{\sqrt{2} m_{t}}\right)\left(1+\frac{\alpha_{s}}{\pi}\left(\frac{11}{4}+\frac{1}{6} \log \left(\frac{\mu^{2}}{m_{t}^{2}}\right)\right)\right)+\mathcal{O}\left(\alpha_{s}^{3}\right), \\
\alpha_{s}^{\mathrm{HEFT}} & =\alpha_{s}, \\
m_{b}^{\mathrm{HEFT}} & =m_{b}+\mathcal{O}\left(\alpha_{s}^{2}\right), \\
y_{b}^{\mathrm{HEFT}} & =y_{b}+y_{t}\left(\frac{\alpha_{s}}{\pi}\right)^{2} \frac{m_{b}}{m_{t}} C_{F}\left(\frac{5}{24}-\frac{1}{4} \log \left(\frac{\mu_{R}^{2}}{m_{t}^{2}}\right)\right)+\mathcal{O}\left(\alpha_{s}^{3}\right) .
\end{aligned}
$$

In our "limited" SM, we can keep the top-quark Yukawa coupling independent from the mass, which is useful to keep track of which terms of the EFT correspond to the expansion of the parts of the SM amplitudes we wish to consider in eq. (2.6). As discussed in refs. [66, 67], there are $\mathcal{O}\left(\alpha_{s}^{2}\right)$ contributions to both the bottom-quark mass and Yukawa coupling, associated to the SM diagrams shown in figures $12 \mathrm{a}$ and $12 \mathrm{~b}$, respectively, and we have to consider only the latter at the perturbative order we are interested in. Indeed, in our SM picture, the bottom Yukawa correction contributes to the NLO QCD cross section of $b \bar{b} H$ production that involves the top-quark Yukawa coupling $\left(y_{t}^{2}\right.$ and $\left.y_{b} y_{t}\right)$, while the mass correction would contribute only to the NNLO QCD cross section proportional to $y_{b}^{2}$, which is beyond the accuracy under consideration. A critical look at these two corrections shows that they are likely to have a similar impact and it might be surprising to include one, but not the other. It turns out, however, that the correction to the HEFT bottom Yukawa is extremely small: it yields a permille effect to the $\overline{\mathrm{MS}}$ bottom-quark Yukawa, which contributes through an already subleading production channel. Thus, we could have ignored this correction to the EFT bottom-quark Yukawa without affecting our 


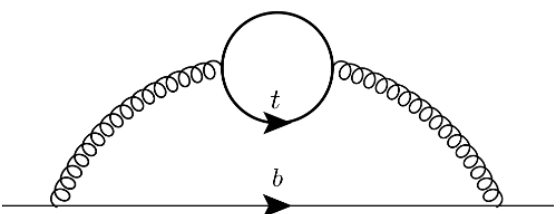

(a)

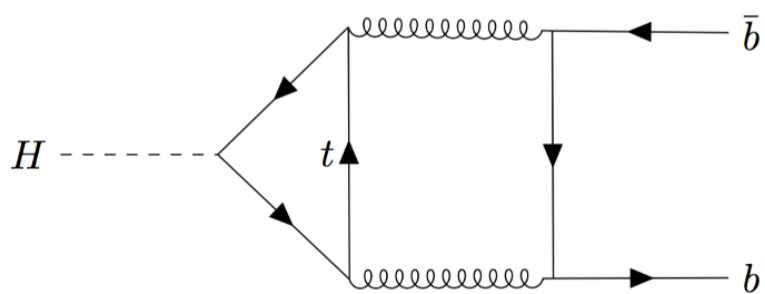

(b)

Figure 12. Standard Model diagrams whose large top mass expansion will yield corrections to (a) the bottom-quark mass and (b) the bottom-quark Yukawa coupling.

phenomenological results. Instead we have chosen to keep it in order to have an exact description of the leading power expansion of the SM amplitude at the perturbative order under consideration. Since the $\mathcal{O}\left(\alpha_{s}^{2}\right)$ corrections to the Yukawa coupling and the mass are of similar numerical size, only a large effect from the bottom Yukawa correction would have been a motivation to include also the mass correction. Since this is not the case, we refrain from including the latter.

\section{The HEFT bottom-quark Yukawa at $\mathcal{O}\left(1 / m_{t}\right)$}

In this appendix, we rederive through a direct calculation the power-suppressed correction to the bottom-quark Yukawa coupling in the HEFT that was first obtained in refs. [66, 67]. This correction is required to compute the complete NLO coefficients $\Delta_{y_{t}^{2}}^{(1)}$ (and $\Delta_{y_{b}^{2}}^{(1)}$ ) in the heavy-top mass approximation. Indeed, as mentioned in section 2.2, the matching of the HEFT to the SM requires not only the introduction of an effective point-like coupling between the Higgs boson and gluons, but also corrections, suppressed by inverse powers of the top-quark mass, to renormalisable couplings. In particular, as we shall see in the following, the bottom-quark Yukawa coupling is modified at $\mathcal{O}\left(1 / m_{t}\right)$ by a term scaling like $y_{t} \alpha_{s}^{2} m_{b} / m_{t}$. We obtain this correction by evaluating a form-factor contribution to the amplitude of the $H \rightarrow b \bar{b}$ decay at $\mathcal{O}\left(y_{t} \alpha_{s}^{2}\right)$, first in the HEFT and then as an expansion in inverse powers of the top-quark mass in the SM, and by matching the two results.

The amplitude for the $H \rightarrow b \bar{b}$ decay can be expressed as follows:

$$
\mathcal{A}(H \rightarrow b \bar{b})=\delta_{i i^{\prime}} \bar{u}_{\sigma}^{h} \hat{\mathcal{A}}_{\sigma \sigma^{\prime}} v_{\sigma^{\prime}}^{h^{\prime}}
$$

where $\bar{u}$ and $v$ are the spinors associated to the bottom and the anti-bottom quarks, respectively, $\sigma$ and $\sigma^{\prime}$ are their spin indices, $h$ and $h^{\prime}$ are their helicities, and $i$ and $i^{\prime}$ are color indices. We consider the following form factor

$$
\begin{aligned}
\mathcal{M} & =\sum_{h h^{\prime}} \delta_{i i^{\prime}} \mathcal{A}(H \rightarrow b \bar{b}) \bar{u}^{h} \cdot v^{h^{\prime}} \\
& =\operatorname{Tr}\left[\left(\not p_{b}+m_{b}\right) \hat{\mathcal{A}}\left(\not p_{\bar{b}}-m_{b}\right)\right],
\end{aligned}
$$

where $p_{b}$ and $p_{\bar{b}}$ denote the bottom and anti-bottom momenta, respectively, and $C_{A}$ is the $\mathrm{SU}(N)$ adjoint Casimir $\left(C_{A}=N\right)$. 


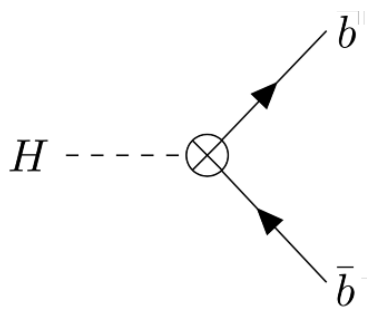

(a)

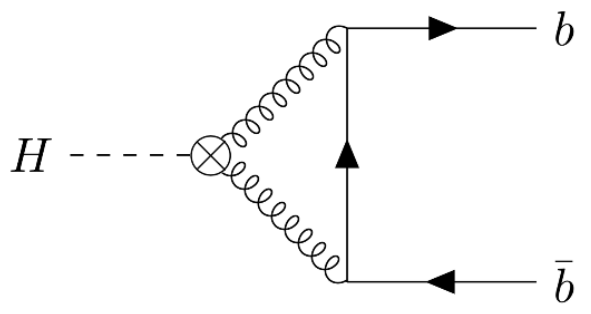

(b)

Figure 13. Diagrams contributing to the $H \rightarrow b \bar{b}$ form factor in the HEFT at $\mathcal{O}\left(y_{t} \alpha_{s}^{2} / m_{t}\right)$. In terms of HEFT couplings, these diagrams scale like $\mathcal{O}\left(\alpha_{s} C_{1}\right)$.

In the HEFT, two types of diagrams contribute to $\mathcal{M}$ at $\mathcal{O}\left(\alpha_{s}^{2} y_{t} / m_{t}\right)$, the contribution to the tree-level diagram from the bottom-quark Yukawa correction $\delta y_{b}$ and the one-loop diagram with an effective Higgs-gluon coupling, shown in figure 13.

The one loop diagram is UV-divergent and is rendered finite by the renormalisation of the bottom-quark Yukawa. Note that we should a priori renormalize the bottom-quark field as well. However, there is no bottom-quark propagator correction that is of order $\mathcal{O}\left(\alpha_{s} C_{1}\right)$. Therefore, the field renormalization counterterm is not relveant in this calculation.

In the $\overline{\mathrm{MS}}$ scheme, we find that we need to define the following counterterm for the HEFT bottom-quark Yukawa:

$$
\frac{\alpha_{s}}{\pi} \sqrt{2} m_{b} C_{F} \frac{3}{4} \frac{C_{1}}{\epsilon}=-y_{t}\left(\frac{\alpha_{s}}{\pi}\right)^{2}\left(\frac{m_{b}}{m_{t}}\right) \frac{C_{F}}{4 \epsilon},
$$

where $C_{F}$ is the fundamental representation Casimir of $\mathrm{SU}(N), \epsilon$ is usual dimensional regularisation parameter in $d=4-2 \epsilon$ spacetime dimensions. Note that the renormalization scheme chosen for other parameters is irrelevant to the matching procedure at hand, since it only has effects beyond accuracy. We can therefore write the following expression for the bare HEFT bottom-quark Yukawa

$$
y_{b}^{\mathrm{HEFT}, B}=y_{b}^{\mathrm{SM}, B}+y_{t}\left(\frac{\alpha_{s}}{\pi}\right)^{2}\left(\frac{m_{b}}{m_{t}}\right)\left[-\frac{C_{F}}{4 \epsilon}+\Delta_{F}\right],
$$

where $\Delta_{F}$ is the finite contribution to $y_{b}^{\mathrm{HEFT}}$ that we seek to obtain. Similarly to the renormalization, we should in principle allow for the possibility that the renormalized bottom-quark field in the HEFT $(\tilde{b})$ is matched to the SM field through a nontrivial finite counterterm. However, as was already pointed out in the previous appendix, no SM bottomquark propagator correction has the correct scaling in the top Yukawa to contribute to the $H \rightarrow b \bar{b}$ amplitude at the order of interest.

We find the following expression for the $\mathcal{O}\left(y_{t} \alpha_{s}^{2}\right)$ part of the renormalised form factor in the Euclidian region $\left(m_{H}^{2}>0\right)$ :

$$
\begin{aligned}
\left.\mathcal{A}_{H \rightarrow b \bar{b}}^{\mathrm{HEFT}}\right|_{y_{t} \alpha_{s}^{2}}= & i y_{t}\left(\frac{\alpha_{s}}{\pi}\right)^{2} C_{A} C_{F}\left(\frac{m_{b}}{m_{t}}\right) m_{b}^{2} \frac{(2 r+1)}{r(r+1) 18 \sqrt{2}}(3 G(0,-1, r)+3 G(0,0, r) \\
& \left.-3 G(-1,-1, r)-3 G(-1,0, r)+9(1+2 r) \log \left(\frac{m_{b}^{2}}{\mu^{2}}\right)-24 r+2 \pi^{2}-12\right) \\
& +i y_{t}\left(\frac{\alpha_{s}}{\pi}\right)^{2}\left(\frac{m_{b}}{m_{t}}\right) C_{A} \frac{m_{b}^{2}}{\sqrt{2}} \frac{(2 r+1)^{2}}{r(r+1)} \Delta_{F},
\end{aligned}
$$



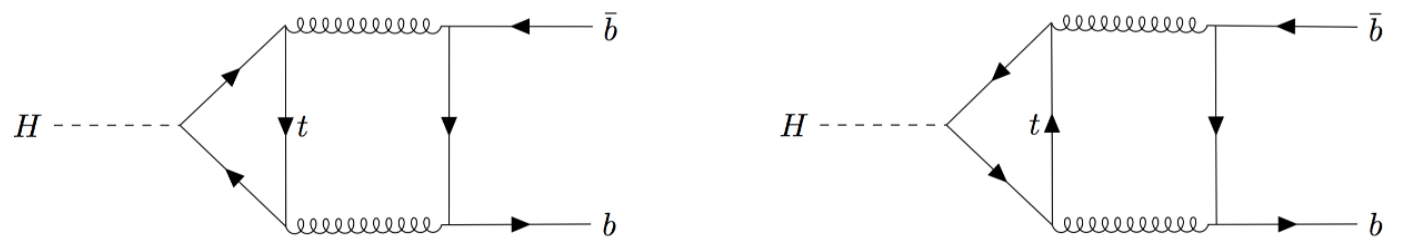

Figure 14. Diagrams contributing to $\mathcal{M}$ at $\mathcal{O}\left(y_{t} \alpha_{s}^{2}\right)$ in the SM.

where $r=\frac{\sqrt{\tau} \sqrt{\tau+4}-\tau}{2 \tau}$, with $\tau=-\frac{m_{H}^{2}}{m_{b}^{2}}$. The form factor is expressed in terms of multiple polylogarithms $G$ [90, 91] defined iteratively by

$$
G\left(a_{1}, \ldots a_{n} ; x\right)=\int_{0}^{x} d t \frac{G\left(a_{2}, \ldots, a_{n} ; t\right)}{t-a_{1}},
$$

where $G(; x)=1$ and we define the special case where all $a_{i}$ are 0 as

$$
G(\underbrace{0, \ldots, 0}_{n} ; x)=\frac{1}{n !} \log ^{n}(x) .
$$

To derive $\Delta_{F}$, we need to compute the heavy-top quark limit (the leading term in $1 / m_{t}$ ) of the SM expression for $\mathcal{M}$. At the perturbative order of interest two two-loop diagrams contribute, shown in figure 14 .

The SM form factor can be evaluated using modern multi-loop calculation techniques. We generate FORM [92] expressions for these form factors using our own QGRAF [93] interface. After the spinor and tensor algebra is performed with FORM, we obtain a scalar expression in terms of kinematic invariants involving the external momenta and the loop momenta. We define the following family of integrals:

$$
J\left(n_{1}, \ldots, n_{7}\right)=\int \frac{d^{d} k_{1}}{(4 \pi)^{d}} \frac{d^{d} k_{2}}{(4 \pi)^{d}} \frac{1}{D_{1}^{n_{1}} \ldots D_{7}^{n_{7}}},
$$

where the denominators are defined as

$$
\begin{array}{lll}
D_{1}=k_{2}^{2}-m_{b}^{2}, & D_{2}=\left(k_{2}-p_{1}\right)^{2}, & D_{3}=\left(k_{2}+p_{2}\right)^{2}, \\
D_{4}=\left(k_{1}-p_{1}\right)^{2}-m_{t}^{2}, & D_{5}=\left(k_{1}+p_{2}\right)^{2}-m_{t}^{2}, & D_{6}=\left(k_{1}-k_{2}\right)^{2}-m_{t}^{2}, \\
D_{7}=\left(k_{1}+k_{2}\right)^{2}-m_{t}^{2} . & &
\end{array}
$$

The form factor is expressed as a linear combination of integrals in this family using MATHEMATICA. We reduce the set of integrals required for the expression of the amplitude to a basis of master integrals using LITERED [94]. We obtain a set of 23 master integrals shown in eq. (C.11), which we need expand in $1 / m_{t}$ :

$$
\begin{aligned}
& J(0,0,0,1,0,1,0), J(1,0,0,1,0,0,0), J(0,0,1,1,0,1,0), J(0,0,2,1,0,1,0), \\
& J(0,0,1,2,0,1,0), J(0,0,0,1,1,1,0), J(0,1,1,1,0,0,0), J(2,0,0,0,1,1,0), \\
& J(1,0,0,1,0,1,0), J(2,0,0,1,0,1,0), J(1,0,0,1,1,0,0), J(0,1,1,1,1,0,0), \\
& J(1,0,0,1,1,1,0), J(2,0,0,1,1,1,0), J(1,0,0,1,1,2,0), J(1,0,0,2,1,1,0), \\
& J(1,0,1,1,0,1,0), J(2,0,1,1,0,1,0), J(1,0,1,2,0,1,0), J(1,1,1,1,0,0,0), \\
& J(0,1,1,1,1,1,0), J(1,1,1,1,1,0,0), J(1,1,1,1,1,1,0) .
\end{aligned}
$$


These master integrals can be expressed in the parametric Feynman representation and their expansion for a heavy top-quark mass ${ }^{10}$ is done using the MATHEMATICA package ASY [95] to perform an expansion by region [96]. The expansion significantly simplifies the integrals and most can readily be identified with combinations of Euler $\Gamma, B$ integrals and logarithms, with the exception of $J(1,1,1,1,1,0,0)$ and $J(1,1,1,1,1,1,0)$. These two integrals can be expressed in terms of multiple polylogarithms and can be straightforwardly evaluated using the algorithm described in appendix $\mathrm{C}$ of [97], whose application is significantly simplified by the package PolylogTools [98] which implements the reduction of multiple polylogarithms to the canonical form defined in ref. [97] and many useful automated tools to integrate multiple polylogarithms in canonical form. The expression for all master integrals in the Euclidean region $\left(m_{H}^{2}<0\right)$ can be found in appendix D. These integrals have been checked by comparing their evaluation with GINAC [99] to the numerical integration provided by FIESTA [100] in the Euclidean region, showing excellent agreement.

By insertion of the expanded master integrals into the form factor and expansion of their coefficients for large top-quark masses, we obtain the expression for the SM version of $\mathcal{M}$ at order $1 / m_{t}{ }^{11}$ The form factor is UV-finite in the SM at this order, and is therefore independent of the choice of the renormalization scheme. We ultimately obtain the following expression for the form factor in the SM:

$$
\begin{aligned}
\mathcal{A}_{\mathrm{SM}}= & i y_{t}\left(\alpha_{s}\right)^{2} C_{A} C_{F}\left(\frac{m_{b}}{m_{t}}\right) \frac{m_{b}^{2}}{36 \sqrt{2} \pi^{2}} \frac{(2 r+1)}{r(r+1)}(6 G(0,-1, r)+6 G(0,0, r) \\
& \left.-6 G(-1,-1, r)-6 G(-1,0, r)+36(2 r+1) \log \left(\frac{m_{b}}{m_{t}}\right)-78 r+4 \pi^{2}-39\right) .
\end{aligned}
$$

In the last step of our calculation we match the form factor in the two theories. We find that the polylogarithmic dependence of the two expressions is exactly the same, leaving us with the condition for the two renormalised expressions to be equal:

$$
\Delta_{F}=\frac{C_{F}}{24}\left(5-6 \log \left(\frac{\mu_{R}^{2}}{m_{t}^{2}}\right)\right)
$$

which is in agreement with the result obtained in refs. $[66,67]$ through low-energy theorems.

\section{Master integrals}

In this appendix, we show the results for the 23 master integrals that appear in the calculation of the two-loop form factor expanded in the infinite top mass limit that we evluated in the previous appendix. The integrals showed here are defined with a $\overline{\mathrm{MS}}$ prefactor

$$
J\left(n_{1}, \ldots, n_{7}\right)=(4 \pi)^{-2 \epsilon} e^{2 \epsilon \gamma} m_{t}^{2 \sum n_{i}-2 d} \int \frac{d^{d} k_{1}}{(2 \pi)^{d}} \frac{d^{d} k_{2}}{(2 \pi)^{d}} \frac{1}{D_{1}^{n_{1}} \ldots D_{7}^{n_{7}}},
$$

\footnotetext{
${ }^{10}$ This is technically achieved by rescaling the invariants with a spurious parameter $\rho$ as $m_{b} \rightarrow \rho m_{b}$ and $m_{H} \rightarrow \rho m_{H}$ and taking the limit $\rho \rightarrow 0$.

${ }^{11}$ We verified that the master integrals were all expanded to a sufficient order in $1 / m_{t}$ by adding dummy higher order terms to their expressions and checking that they vanish in the $1 / m_{t}$ term of the form factor.
} 
where

$$
\begin{array}{lll}
D_{1}=k_{2}^{2}-m_{b}^{2}, & D_{2}=\left(k_{2}-p_{1}\right)^{2}, & D_{3}=\left(k_{2}+p_{2}\right)^{2}, \\
D_{4}=\left(k_{1}-p_{1}\right)^{2}-m_{t}^{2}, & D_{5}=\left(k_{1}+p_{2}\right)^{2}-m_{t}^{2}, & D_{6}=\left(k_{1}-k_{2}\right)^{2}-m_{t}^{2}, \\
D_{7}=\left(k_{1}+k_{2}\right)^{2}-m_{t}^{2} . & &
\end{array}
$$

We express our integrals in terms of $t=m_{b}^{2} / m_{t}^{2}$ and $r=\frac{\sqrt{\tau} \sqrt{\tau+4}-\tau}{2 \tau}$, with $\tau=-\frac{m_{H}^{2}}{m_{b}^{2}}$.

$$
\begin{aligned}
& J(0,0,0,1,0,1,0)=\left(-\frac{1}{256 \pi^{4}}\right) \frac{1}{\epsilon^{2}}+\left(-\frac{1}{128 \pi^{4}}\right) \frac{1}{\epsilon}-\frac{1}{1536 \pi^{2}}-\frac{3}{256 \pi^{4}} \\
& J(1,0,0,1,0,0,0)=\frac{1}{\epsilon^{2}}\left(t\left(-\frac{1}{256 \pi^{4}}\right)\right)+\frac{1}{\epsilon}\left(t \frac{\log (t)-2}{256 \pi^{4}}\right)+t\left(-\frac{3 \log ^{2}(t)-12 \log (t)+\pi^{2}+18}{1536 \pi^{4}}\right) \\
& J(0,0,1,1,0,1,0)=\left(-\frac{1}{256 \pi^{4}}\right) \frac{1}{\epsilon^{2}}+\frac{1}{\epsilon}\left(t\left(-\frac{1}{1024 \pi^{4} r(r+1)}\right)-\frac{3}{256 \pi^{4}}\right) \\
& \quad+\frac{1}{9216 \pi^{4} r^{2}(r+1)^{2}} t^{2}+\frac{1}{2048 \pi^{4} r(r+1)} t-\frac{1}{1536 \pi^{2}}-\frac{7}{256 \pi^{4}} \\
& J(0,0,2,1,0,1,0)=\frac{1}{512 \pi^{4}} \frac{1}{\epsilon^{2}}+\frac{1}{\epsilon}\left(t^{2} \frac{1}{15360 \pi^{4} r^{2}(r+1)^{2}}+t\left(-\frac{1}{1536 \pi^{4} r(r+1)}\right)-\frac{1}{512 \pi^{4}}\right) \\
& \quad+\frac{17}{115200 \pi^{4} r^{2}(r+1)^{2}} t^{2}+\frac{1}{9216 \pi^{4} r(r+1)} t+\frac{18+\pi^{2}}{3072 \pi^{4}} \\
& J(0,0,1,2,0,1,0)=\left(-\frac{1}{512 \pi^{4}}\right) \frac{1}{\epsilon^{2}}+\left(-\frac{1}{512 \pi^{4}}\right) \frac{1}{\epsilon}+\left(-\frac{1}{18432 \pi^{4} r^{2}(r+1)^{2}}\right) t^{2} \\
& \quad+\frac{1}{1024 \pi^{4} r(r+1)} t+\frac{-6-\pi^{2}}{3072 \pi^{4}} \\
& J(0,0,0,1,1,1,0)=\left(-\frac{1}{256 \pi^{4}}\right) \frac{1}{\epsilon^{2}}+\frac{1}{\epsilon}\left(t^{2}\left(-\frac{1}{15360 \pi^{4} r^{2}(r+1)^{2}}\right)+t \frac{1}{1536 \pi^{4} r(r+1)}-\frac{1}{256 \pi^{4}}\right) \\
& \quad+\left(-\frac{1}{7680 \pi^{4} r^{2}(r+1)^{2}}\right) t^{2}+\frac{1}{1536 \pi^{4} r(r+1)} t+\frac{-6-\pi^{2}}{1536 \pi^{4}} \\
& J(0,1,1,1,0,0,0)=\left(-\frac{1}{256 \pi^{4}}\right) \frac{1}{\epsilon^{2}}+\frac{1}{\epsilon} \frac{\log (r(r+1))+\log (t)-3}{256 \pi^{4}} \\
& \left.\quad+20 g(r(r+1)) \log (t)-10{ }^{2}(r+1)\right)-6 \log (r(r+1))-\log (t)+6 \log (t)-14
\end{aligned}
$$$$
+\frac{2 \log (r(r+1)) \log (t)-\log ^{2}(r(r+1))-6 \log (r(r+1))-\log ^{2}(t)+6 \log (t)-14}{512 \pi^{4}}
$$$$
J(2,0,0,0,1,1,0)=\left(-\frac{1}{512 \pi^{4}}\right) \frac{1}{\epsilon^{2}}+\frac{1}{\epsilon} \frac{2 \log (t)-1}{512 \pi^{4}}+t^{2} \frac{6 \log (t)-5}{9216 \pi^{4}}
$$$$
+\frac{-6 \log ^{2}(t)-\pi^{2}+18}{3072 \pi^{4}}+t \frac{2 \log (t)-3}{1024 \pi^{4}}
$$$$
J(1,0,0,1,0,1,0)=\frac{1}{\epsilon^{2}}\left(t\left(-\frac{1}{512 \pi^{4}}\right)-\frac{1}{256 \pi^{4}}\right)+\frac{1}{\epsilon}\left(t^{3}\left(-\frac{1}{3072 \pi^{4}}\right)+t \frac{4 \log (t)-5}{1024 \pi^{4}}-\frac{3}{256 \pi^{4}}\right)
$$$$
+t^{3} \frac{10 \log (t)-21}{30720 \pi^{4}}+t^{2} \frac{6 \log (t)-11}{4608 \pi^{4}}+t \frac{-12 \log ^{2}(t)+24 \log (t)-2 \pi^{2}+9}{6144 \pi^{4}}+\frac{-42-\pi^{2}}{1536 \pi^{4}}
$$$$
J(2,0,0,1,0,1,0)=\left(-\frac{1}{512 \pi^{4}}\right) \frac{1}{\epsilon^{2}}+\frac{1}{\epsilon} \frac{2 \log (t)-1}{512 \pi^{4}}+t^{2} \frac{6 \log (t)-5}{9216 \pi^{4}}
$$$$
+\frac{-6 \log ^{2}(t)-\pi^{2}+18}{3072 \pi^{4}}+t \frac{2 \log (t)-3}{1024 \pi^{4}}
$$ 


$$
\begin{aligned}
& J(1,0,0,1,1,0,0)=\frac{1}{\epsilon^{2}}\left(t\left(-\frac{1}{256 \pi^{4}}\right)\right) \\
& +\frac{1}{\epsilon}\left(t^{3}\left(-\frac{1}{15360 \pi^{4} r^{2}(r+1)^{2}}\right)+t^{2} \frac{1}{1536 \pi^{4} r(r+1)}+t \frac{\log (t)-1}{256 \pi^{4}}\right) \\
& +t^{3} \frac{\log (t)-2}{15360 \pi^{4} r^{2}(r+1)^{2}}+t^{2}\left(-\frac{\log (t)-1}{1536 \pi^{4} r(r+1)}\right)+t\left(-\frac{3 \log ^{2}(t)-6 \log (t)+\pi^{2}+6}{1536 \pi^{4}}\right) \\
& J(0,1,1,1,1,0,0)=\left(-\frac{1}{256 \pi^{4}}\right) \frac{1}{\epsilon^{2}}+\frac{1}{\epsilon}\left(\frac{-\log (r(r+1))+\log (t)-2}{256 \pi^{4}}\right. \\
& \left.+t^{2}\left(-\frac{1}{15360 \pi^{4} r^{2}(r+1)^{2}}\right)+t \frac{1}{1536 \pi^{4} r(r+1)}\right)+t^{2} \frac{-\log (r(r+1))+\log (t)-3}{15360 \pi^{4} r^{2}(r+1)^{2}} \\
& +\frac{2 \log (r(r+1)) \log (t)-\log ^{2}(r(r+1))-4 \log (r(r+1))-\log ^{2}(t)+4 \log (t)-8}{512 \pi^{4}} \\
& +t \frac{\log (r(r+1))-\log (t)+2}{1536 \pi^{4} r(r+1)} \\
& J(1,0,0,1,1,1,0)=\left(-\frac{1}{512 \pi^{4}}\right) \frac{1}{\epsilon^{2}}+\frac{1}{\epsilon}\left(t^{2}\left(-\frac{1}{15360 \pi^{4} r^{2}(r+1)^{2}}\right)+t \frac{1}{1536 \pi^{4} r(r+1)}\right. \\
& \left.+t^{3} \frac{30 r^{4}+60 r^{3}+23 r^{2}-7 r+2}{92160 \pi^{4} r^{2}(r+1)^{2}}-\frac{1}{512 \pi^{4}}\right)+t \frac{18 r^{2}-12(r+1) \log (t) r+18 r-1}{6144 \pi^{4} r(r+1)} \\
& +t^{3} \frac{78 r^{4}+156 r^{3}+61 r^{2}-17 r-\left(30 r^{4}+60 r^{3}+23 r^{2}-7 r+2\right) \log (t)+5}{92160 \pi^{4} r^{2}(r+1)^{2}} \\
& +t^{2} \frac{300 r^{4}+600 r^{3}+215 r^{2}-90\left(4 r^{3}+8 r^{2}+3 r-1\right) \log (t) r-85 r-43}{552960 \pi^{4} r^{2}(r+1)^{2}}-\frac{1}{3072 \pi^{2}}-\frac{1}{512 \pi^{4}} \\
& J(2,0,0,1,1,1,0)=t\left(-\frac{\left(6 r^{2}+6 r-1\right)(2 \log (t)-1)}{12288 \pi^{4} r(r+1)}\right) \\
& +t^{2}\left(-\frac{\left(30 r^{4}+60 r^{3}+25 r^{2}-5 r+1\right)(3 \log (t)-1)}{138240 \pi^{4} r^{2}(r+1)^{2}}\right)-\frac{\log (t)}{512 \pi^{4}}+\frac{1}{512 \pi^{4}} \\
& J(1,0,0,1,1,2,0)=\frac{1}{\epsilon}\left(t^{3}\left(-\frac{90 r^{4}+180 r^{3}+77 r^{2}-13 r+2}{215040 \pi^{4} r^{2}(r+1)^{2}}\right)\right) \\
& +t\left(-\frac{2 r^{2}-12(r+1) \log (t) r+2 r+5}{18432 \pi^{4} r(r+1)}\right) \\
& +t^{3}\left(-\frac{792 r^{4}+1584 r^{3}+686 r^{2}-106 r-3\left(90 r^{4}+180 r^{3}+77 r^{2}-13 r+2\right) \log (t)+17}{645120 \pi^{4} r^{2}(r+1)^{2}}\right) \\
& +t^{2}\left(-\frac{168 r^{4}+336 r^{3}+172 r^{2}-90\left(8 r^{3}+16 r^{2}+7 r-1\right) \log (t) r+4 r-47}{1382400 \pi^{4} r^{2}(r+1)^{2}}\right)+\frac{1}{256 \pi^{4}} \\
& J(1,0,0,2,1,1,0)=\frac{1}{\epsilon}\left(t^{2} \frac{1}{15360 \pi^{4} r^{2}(r+1)^{2}}+t\left(-\frac{1}{3072 \pi^{4} r(r+1)}\right)\right. \\
& \left.+t^{3}\left(-\frac{10 r^{4}+20 r^{3}+7 r^{2}-3 r+1}{35840 \pi^{4} r^{2}(r+1)^{2}}\right)+\frac{1}{512 \pi^{4}}\right)+t \frac{3 r(r+1) \log (t)-2\left(r^{2}+r+1\right)}{4608 \pi^{4} r(r+1)} \\
& +t^{3}\left(-\frac{176 r^{4}+352 r^{3}+126 r^{2}-50 r-6\left(10 r^{4}+20 r^{3}+7 r^{2}-3 r+1\right) \log (t)+17}{215040 \pi^{4} r^{2}(r+1)^{2}}\right) \\
& +t^{2} \frac{-36 r^{4}-72 r^{3}-19 r^{2}+30\left(3 r^{3}+6 r^{2}+2 r-1\right) \log (t) r+17 r+29}{230400 \pi^{4} r^{2}(r+1)^{2}}
\end{aligned}
$$




$$
\begin{aligned}
& J(1,0,1,1,0,1,0)=\left(-\frac{1}{512 \pi^{4}}\right) \frac{1}{\epsilon^{2}}+\frac{1}{\epsilon}\left(\frac{\log (t)}{256 \pi^{4}}-\frac{5}{512 \pi^{4}}\right) \\
& +t \frac{-26 r^{2}-26 r+6\left(2 r^{2}+2 r-1\right) \log (t)+19}{18432 \pi^{4} r(r+1)} \\
& +t^{2}\left(-\frac{37 r^{4}+74 r^{3}+23 r^{2}-14 r-5\left(6 r^{4}+12 r^{3}+4 r^{2}-2 r+1\right) \log (t)+12}{230400 \pi^{4} r^{2}(r+1)^{2}}\right) \\
& -\frac{\log ^{2}(t)}{512 \pi^{4}}+\frac{\log (t)}{128 \pi^{4}}-\frac{1}{3072 \pi^{2}}-\frac{5}{512 \pi^{4}} \\
& J(2,0,1,1,0,1,0)=\frac{1}{\epsilon^{2}}\left(\frac{1}{t}\left(-\frac{1}{512 \pi^{4}}\right)\right) \\
& +\frac{1}{\epsilon}\left(t\left(-\frac{1}{30720 \pi^{4} r^{2}(r+1)^{2}}\right)+t^{2} \frac{10 r^{4}+20 r^{3}+7 r^{2}-3 r+1}{107520 \pi^{4} r^{2}(r+1)^{2}}+\frac{1}{t} \frac{\log (t)}{512 \pi^{4}}+\frac{1}{3072 \pi^{4} r(r+1)}\right) \\
& +\frac{3110 r^{4}+6220 r^{3}+1967 r^{2}-1143 r+486}{22579200 \pi^{4} r^{2}(r+1)^{2}} t^{2} \\
& +t\left(-\frac{81 r^{4}+162 r^{3}+49 r^{2}-32 r-15\left(6 r^{4}+12 r^{3}+4 r^{2}-2 r+1\right) \log (t)+60}{460800 \pi^{4} r^{2}(r+1)^{2}}\right) \\
& -\frac{\log (t)}{3072 \pi^{4} r(r+1)}+\frac{1}{1536 \pi^{4} r(r+1)}+\frac{1}{t}\left(-\frac{3 \log ^{2}(t)+\pi^{2}}{3072 \pi^{4}}\right) \frac{\log (t)}{1536 \pi^{4}}-\frac{5}{4608 \pi^{4}} \\
& J(1,0,1,2,0,1,0)=t \frac{14 r^{2}+14 r-6\left(2 r^{2}+2 r-1\right) \log (t)-13}{36864 \pi^{4} r(r+1)} \\
& +t^{2} \frac{44 r^{4}+88 r^{3}+26 r^{2}-18 r-10\left(6 r^{4}+12 r^{3}+4 r^{2}-2 r+1\right) \log (t)+19}{460800 \pi^{4} r^{2}(r+1)^{2}} \\
& -\frac{\log (t)}{512 \pi^{4}}+\frac{3}{512 \pi^{4}} \\
& J(1,1,1,1,0,0,0)= \\
& \frac{1}{\epsilon}\left(\frac{1}{t} \frac{r(r+1)\left(-3 G(-1,-1, r)-3 G(-1,0, r)+3 G(0,-1, r)+3 G(0,0, r)+2 \pi^{2}\right)}{768 \pi^{4}(2 r+1)}\right) \\
& +\frac{1}{t} \frac{1}{1536 \pi^{4}(2 r+1)}\left(r ( r + 1 ) \left(\pi^{2} G(-1, r)+8 \pi^{2} G\left(-\frac{1}{2}, r\right)-\pi^{2} G(0, r)-6 G(-1,-1, r)\right.\right. \\
& -6 G(-1,0, r)+6 G(0,-1, r)+6 G(0,0, r)-6 G(-1,-1,-1, r) \\
& -6 G(-1,-1,0, r)-6 G(-1,0,-1, r)-6 G(-1,0,0, r) \\
& -12 G\left(-\frac{1}{2},-1,-1, r\right)-12 G\left(-\frac{1}{2},-1,0, r\right)+12 G\left(-\frac{1}{2}, 0,-1, r\right)+12 G\left(-\frac{1}{2}, 0,0, r\right) \\
& +6 G(0,-1,-1, r)+6 G(0,-1,0, r)+6 G(0,0,-1, r)+6 G(0,0,0, r)+6 G(-1,-1, r) \log (t) \\
& \left.\left.+6 G(-1,0, r) \log (t)-6 G(0,-1, r) \log (t)-6 G(0,0, r) \log (t)-4 \pi^{2} \log (t)+30 \zeta(3)+4 \pi^{2}\right)\right) \\
& J(0,1,1,1,1,1,0)=t^{2} \frac{\log (r(r+1))-\log (t)+1}{46080 \pi^{4} r^{2}(r+1)^{2}}+t \frac{-2 \log (r(r+1))+2 \log (t)-3}{12288 \pi^{4} r(r+1)} \\
& +\frac{\log (r(r+1))}{512 \pi^{4}}-\frac{\log (t)}{512 \pi^{4}}+\frac{3}{512 \pi^{4}}
\end{aligned}
$$




$$
\begin{aligned}
& J(1,1,1,1,1,0,0)= \\
& \frac{1}{\epsilon}\left(\frac{1}{t} \frac{r(r+1)\left(-3 G(-1,-1, r)-3 G(-1,0, r)+3 G(0,-1, r)+3 G(0,0, r)+2 \pi^{2}\right)}{768 \pi^{4}(2 r+1)}\right) \\
& +\frac{1}{t} \frac{1}{1536 \pi^{4}(2 r+1)}\left(r ( r + 1 ) \left(\pi^{2} G(-1, r)+8 \pi^{2} G\left(-\frac{1}{2}, r\right)-\pi^{2} G(0, r)\right.\right. \\
& -6 G(-1,-1,-1, r)-6 G(-1,-1,0, r)-6 G(-1,0,-1, r)-6 G(-1,0,0, r)-12 G\left(-\frac{1}{2},-1,-1, r\right) \\
& -12 G\left(-\frac{1}{2},-1,0, r\right)+12 G\left(-\frac{1}{2}, 0,-1, r\right)+12 G\left(-\frac{1}{2}, 0,0, r\right)+6 G(0,-1,-1, r) \\
& +6 G(0,-1,0, r)+6 G(0,0,-1, r)+6 G(0,0,0, r)+6 G(-1,-1, r) \log (t) \\
& \left.\left.+6 G(-1,0, r) \log (t)-6 G(0,-1, r) \log (t)-6 G(0,0, r) \log (t)-4 \pi^{2} \log (t)+30 \zeta(3)\right)\right) \\
& +t \frac{-3 G(-1,-1, r)-3 G(-1,0, r)+3 G(0,-1, r)+3 G(0,0, r)+2 \pi^{2}}{46080 \pi^{4} r(r+1)(2 r+1)} \\
& +\frac{r G(-1,-1, r)}{1536 \pi^{4}(r+1)(2 r+1)}+\frac{r G(-1,0, r)}{1536 \pi^{4}(r+1)(2 r+1)}-\frac{r G(0,-1, r)}{1536 \pi^{4}(r+1)(2 r+1)} \\
& -\frac{r G(0,0, r)}{1536 \pi^{4}(r+1)(2 r+1)}+\frac{G(-1,-1, r)}{1536 \pi^{4}(r+1)(2 r+1)} \\
& +\frac{G(-1,0, r)}{1536 \pi^{4}(r+1)(2 r+1)}-\frac{G(0,-1, r)}{1536 \pi^{4}(r+1)(2 r+1)}-\frac{G(0,0, r)}{1536 \pi^{4}(r+1)(2 r+1)} \\
& -\frac{r}{2304 \pi^{2}(r+1)(2 r+1)}-\frac{1}{2304 \pi^{2}(r+1)(2 r+1)} \\
& J(1,1,1,1,1,1,0)=\frac{1}{\epsilon}\left(t\left(-\frac{r^{2}+r-1}{15360 \pi^{4} r(r+1)}\right)\right) \\
& +\frac{1}{t}\left(-\frac{r(r+1)\left(-3 G(-1,-1, r)-3 G(-1,0, r)+3 G(0,-1, r)+3 G(0,0, r)+2 \pi^{2}\right)}{1536 \pi^{4}(2 r+1)}\right) \\
& -\frac{G(-1,-1, r)}{6144 \pi^{4}(2 r+1)}-\frac{G(-1,0, r)}{6144 \pi^{4}(2 r+1)}+\frac{G(0,-1, r)}{6144 \pi^{4}(2 r+1)}+\frac{G(0,0, r)}{6144 \pi^{4}(2 r+1)} \\
& +\frac{-86 r^{2}-86 r+91}{921600 \pi^{4} r(r+1)} t-\frac{r \log (t)}{1536 \pi^{4}(2 r+1)}-\frac{\log (t)}{3072 \pi^{4}(2 r+1)}+\frac{1}{9216 \pi^{2}(2 r+1)}+\frac{1}{1152 \pi^{4}}
\end{aligned}
$$

Open Access. This article is distributed under the terms of the Creative Commons Attribution License (CC-BY 4.0), which permits any use, distribution and reproduction in any medium, provided the original author(s) and source are credited.

\section{References}

[1] ATLAS collaboration, Observation of a new particle in the search for the Standard Model Higgs boson with the ATLAS detector at the LHC, Phys. Lett. B 716 (2012) 1 [arXiv: 1207.7214] [INSPIRE].

[2] CMS collaboration, Observation of a New Boson at a Mass of $125 \mathrm{GeV}$ with the CMS Experiment at the LHC, Phys. Lett. B 716 (2012) 30 [arXiv:1207.7235] [InSPIRE].

[3] F. Englert and R. Brout, Broken Symmetry and the Mass of Gauge Vector Mesons, Phys. Rev. Lett. 13 (1964) 321 [INSPIRE]. 
[4] P.W. Higgs, Broken Symmetries and the Masses of Gauge Bosons, Phys. Rev. Lett. 13 (1964) 508 [inSPIRE].

[5] S. Weinberg, A Model of Leptons, Phys. Rev. Lett. 19 (1967) 1264 [inSPIRE].

[6] ATLAS collaboration, Measurements of the Higgs boson production and decay rates and coupling strengths using pp collision data at $\sqrt{s}=7$ and $8 \mathrm{TeV}$ in the ATLAS experiment, Eur. Phys. J. C 76 (2016) 6 [arXiv:1507.04548] [inSPIRE].

[7] ATLAS and CMS collaborations, Combined Measurement of the Higgs Boson Mass in pp Collisions at $\sqrt{s}=7$ and $8 \mathrm{TeV}$ with the ATLAS and CMS Experiments, Phys. Rev. Lett. 114 (2015) 191803 [arXiv: 1503.07589] [INSPIRE].

[8] ATLAS and CMS collaborations, Measurements of the Higgs boson production and decay rates and constraints on its couplings from a combined ATLAS and CMS analysis of the LHC pp collision data at $\sqrt{s}=7$ and 8 TeV, JHEP 08 (2016) 045 [arXiv:1606.02266] [INSPIRE].

[9] ATLAS collaboration, Combined measurements of Higgs boson production and decay using up to $80 \mathrm{fb}^{-1}$ of proton-proton collision data at $\sqrt{\mathrm{s}}=13 \mathrm{TeV}$ collected with the ATLAS experiment, ATLAS-CONF-2019-005.

[10] CMS collaboration, Combined measurement and interpretation of differential Higgs boson production cross sections at $\sqrt{s}=13 \mathrm{TeV}$, CMS-PAS-HIG-17-028.

[11] CMS collaboration, Observation of $t \bar{t} H$ production, Phys. Rev. Lett. 120 (2018) 231801 [arXiv: 1804.02610] [INSPIRE].

[12] ATLAS collaboration, Observation of Higgs boson production in association with a top quark pair at the LHC with the ATLAS detector, Phys. Lett. B 784 (2018) 173 [arXiv: 1806.00425] [INSPIRE].

[13] ATLAS collaboration, Evidence for the Higgs-boson Yukawa coupling to tau leptons with the ATLAS detector, JHEP 04 (2015) 117 [arXiv:1501.04943] [INSPIRE].

[14] CMS collaboration, Observation of the Higgs boson decay to a pair of $\tau$ leptons with the CMS detector, Phys. Lett. B 779 (2018) 283 [arXiv:1708.00373] [INSPIRE].

[15] ATLAS collaboration, Observation of $H \rightarrow b \bar{b}$ decays and $V H$ production with the ATLAS detector, ATLAS-CONF-2018-036.

[16] CMS collaboration, Search for the standard model Higgs boson produced through vector

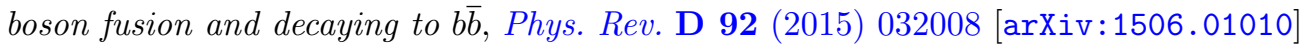
[INSPIRE].

[17] ATLAS collaboration, Search for the Standard Model Higgs boson produced by vector-boson fusion and decaying to bottom quarks in $\sqrt{s}=8 \mathrm{TeV}$ pp collisions with the ATLAS detector, JHEP 11 (2016) 112 [arXiv:1606.02181] [INSPIRE].

[18] CMS collaboration, Search for the standard model Higgs boson produced in association with $a$ W or a $Z$ boson and decaying to bottom quarks, Phys. Rev. D 89 (2014) 012003 [arXiv: 1310.3687] [INSPIRE].

[19] H. Mantler and M. Wiesemann, Top- and bottom-mass effects in hadronic Higgs production at small transverse momenta through LO+NLL, Eur. Phys. J. C 73 (2013) 2467 [arXiv: 1210.8263] [INSPIRE]. 
[20] M. Grazzini and H. Sargsyan, Heavy-quark mass effects in Higgs boson production at the LHC, JHEP 09 (2013) 129 [arXiv: 1306.4581] [INSPIRE].

[21] A. Banfi, P.F. Monni and G. Zanderighi, Quark masses in Higgs production with a jet veto, JHEP 01 (2014) 097 [arXiv:1308.4634] [INSPIRE].

[22] E. Bagnaschi, G. Degrassi, P. Slavich and A. Vicini, Higgs production via gluon fusion in the POWHEG approach in the SM and in the MSSM, JHEP 02 (2012) 088 [arXiv:1111.2854] [INSPIRE].

[23] R. Frederix, S. Frixione, E. Vryonidou and M. Wiesemann, Heavy-quark mass effects in Higgs plus jets production, JHEP 08 (2016) 006 [arXiv:1604.03017] [INSPIRE].

[24] E. Bagnaschi, R.V. Harlander, H. Mantler, A. Vicini and M. Wiesemann, Resummation ambiguities in the Higgs transverse-momentum spectrum in the Standard Model and beyond, JHEP 01 (2016) 090 [arXiv:1510.08850] [INSPIRE].

[25] H. Mantler and M. Wiesemann, Hadronic Higgs production through NLO+PS in the SM, the 2HDM and the MSSM, Eur. Phys. J. C 75 (2015) 257 [arXiv:1504.06625] [INSPIRE].

[26] R.V. Harlander, H. Mantler and M. Wiesemann, Transverse momentum resummation for Higgs production via gluon fusion in the MSSM, JHEP 11 (2014) 116 [arXiv:1409.0531] [INSPIRE].

[27] R. Raitio and W.W. Wada, Higgs Boson Production at Large Transverse Momentum in QCD, Phys. Rev. D 19 (1979) 941 [inSPIRE].

[28] D.L. Rainwater, M. Spira and D. Zeppenfeld, Higgs boson production at hadron colliders: Signal and background processes, in Physics at TeV colliders. Proceedings, Euro Summer School, Les Houches, France, May 21-June 1, 2001 (2002) [hep-ph/0203187] [INSPIRE].

[29] S. Dittmaier, M. Krämer and M. Spira, Higgs radiation off bottom quarks at the Tevatron and the CERN LHC, Phys. Rev. D 70 (2004) 074010 [hep-ph/0309204] [inSPIRE].

[30] M. Wiesemann, R. Frederix, S. Frixione, V. Hirschi, F. Maltoni and P. Torrielli, Higgs production in association with bottom quarks, JHEP 02 (2015) 132 [arXiv:1409.5301] [INSPIRE].

[31] S. Dawson, C.B. Jackson, L. Reina and D. Wackeroth, Exclusive Higgs boson production with bottom quarks at hadron colliders, Phys. Rev. D 69 (2004) 074027 [hep-ph/0311067] [INSPIRE].

[32] S. Dawson, C.B. Jackson, L. Reina and D. Wackeroth, Higgs production in association with bottom quarks at hadron colliders, Mod. Phys. Lett. A 21 (2006) 89 [hep-ph/0508293] [INSPIRE].

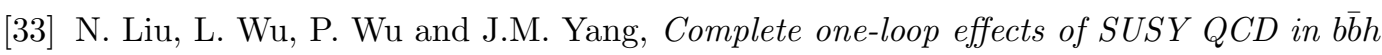
production at the LHC under current experimental constraints, JHEP 01 (2013) 161 [arXiv: 1208.3413] [INSPIRE].

[34] S. Dittmaier, P. Häfliger, M. Krämer, M. Spira and M. Walser, Neutral MSSM Higgs-boson production with heavy quarks: NLO supersymmetric QCD corrections, Phys. Rev. D 90 (2014) 035010 [arXiv:1406.5307] [InSPIRE].

[35] Y. Zhang, NLO electroweak effects on the Higgs boson production in association with a bottom quark pair at the LHC, Phys. Rev. D 96 (2017) 113009 [arXiv:1708.08790] [INSPIRE]. 
[36] D. Dicus, T. Stelzer, Z. Sullivan and S. Willenbrock, Higgs boson production in association with bottom quarks at next-to-leading order, Phys. Rev. D 59 (1999) 094016 [hep-ph/9811492] [INSPIRE].

[37] C. Balázs, H.-J. He and C.P. Yuan, QCD corrections to scalar production via heavy quark fusion at hadron colliders, Phys. Rev. D 60 (1999) 114001 [hep-ph/9812263] [INSPIRE].

[38] R.V. Harlander and W.B. Kilgore, Higgs boson production in bottom quark fusion at next-to-next-to leading order, Phys. Rev. D 68 (2003) 013001 [hep-ph/0304035] [INSPIRE].

[39] J.M. Campbell, R.K. Ellis, F. Maltoni and S. Willenbrock, Higgs-Boson production in association with a single bottom quark, Phys. Rev. D 67 (2003) 095002 [hep-ph/0204093] [INSPIRE].

[40] R.V. Harlander, K.J. Ozeren and M. Wiesemann, Higgs plus jet production in bottom quark annihilation at next-to-leading order, Phys. Lett. B 693 (2010) 269 [arXiv:1007.5411] [INSPIRE].

[41] R. Harlander and M. Wiesemann, Jet-veto in bottom-quark induced Higgs production at next-to-next-to-leading order, JHEP 04 (2012) 066 [arXiv:1111.2182] [INSPIRE].

[42] S. Bühler, F. Herzog, A. Lazopoulos and R. Müller, The fully differential hadronic production of a Higgs boson via bottom quark fusion at NNLO, JHEP 07 (2012) 115 [arXiv: 1204.4415] [INSPIRE].

[43] K.J. Ozeren, Analytic Results for Higgs Production in Bottom Fusion, JHEP 11 (2010) 084 [arXiv: 1010.2977] [INSPIRE].

[44] A. Belyaev, P.M. Nadolsky and C.P. Yuan, Transverse momentum resummation for Higgs

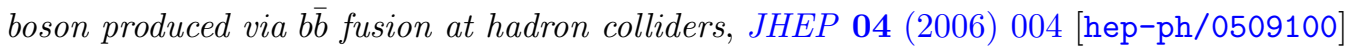
[INSPIRE].

[45] R.V. Harlander, A. Tripathi and M. Wiesemann, Higgs production in bottom quark annihilation: Transverse momentum distribution at NNLO+NNLL, Phys. Rev. D 90 (2014) 015017 [arXiv: 1403.7196] [INSPIRE].

[46] T. Ahmed, M. Mahakhud, P. Mathews, N. Rana and V. Ravindran, Two-loop QCD corrections to Higgs $\rightarrow b+\bar{b}+g$ amplitude, JHEP 08 (2014) 075 [arXiv:1405.2324] [INSPIRE].

[47] T. Gehrmann and D. Kara, The Hbb form factor to three loops in QCD, JHEP 09 (2014) 174 [arXiv: 1407.8114] [INSPIRE].

[48] B. Jager, L. Reina and D. Wackeroth, Higgs boson production in association with $b$ jets in the POWHEG BOX, Phys. Rev. D 93 (2016) 014030 [arXiv:1509.05843] [INSPIRE].

[49] F. Krauss, D. Napoletano and S. Schumann, Simulating b-associated production of $Z$ and Higgs bosons with the SHERPA event generator, Phys. Rev. D 95 (2017) 036012 [arXiv: 1612.04640] [INSPIRE].

[50] F. Maltoni, G. Ridolfi and M. Ubiali, b-initiated processes at the LHC: a reappraisal, JHEP 07 (2012) 022 [Erratum ibid. 04 (2013) 095] [arXiv: 1203.6393] [INSPIRE].

[51] M. Lim, F. Maltoni, G. Ridolfi and M. Ubiali, Anatomy of double heavy-quark initiated processes, JHEP 09 (2016) 132 [arXiv:1605.09411] [INSPIRE].

[52] S. Forte, D. Napoletano and M. Ubiali, Higgs production in bottom-quark fusion in a matched scheme, Phys. Lett. B 751 (2015) 331 [arXiv:1508.01529] [INSPIRE]. 
[53] S. Forte, D. Napoletano and M. Ubiali, Higgs production in bottom-quark fusion: matching beyond leading order, Phys. Lett. B 763 (2016) 190 [arXiv:1607.00389] [INSPIRE].

[54] M. Bonvini, A.S. Papanastasiou and F.J. Tackmann, Resummation and matching of b-quark mass effects in $b \bar{b} H$ production, JHEP 11 (2015) 196 [arXiv:1508.03288] [INSPIRE].

[55] M. Bonvini, A.S. Papanastasiou and F.J. Tackmann, Matched predictions for the $b \bar{b} H$ cross section at the $13 \mathrm{TeV}$ LHC, JHEP 10 (2016) 053 [arXiv:1605.01733] [INSPIRE].

[56] C. Anastasiou and K. Melnikov, Higgs boson production at hadron colliders in NNLO QCD, Nucl. Phys. B 646 (2002) 220 [hep-ph/0207004] [INSPIRE].

[57] C. Anastasiou, C. Duhr, F. Dulat, F. Herzog and B. Mistlberger, Higgs Boson Gluon-Fusion Production in QCD at Three Loops, Phys. Rev. Lett. 114 (2015) 212001 [arXiv: 1503.06056] [INSPIRE].

[58] M. Gerlach, F. Herren and M. Steinhauser, Wilson coefficients for Higgs boson production and decoupling relations to $\mathcal{O}\left(\alpha_{s}^{4}\right)$, JHEP 11 (2018) 141 [arXiv: 1809.06787] [INSPIRE].

[59] B. Mistlberger, Higgs boson production at hadron colliders at $N^{3} L O$ in $Q C D$, JHEP 05 (2018) 028 [arXiv: 1802.00833] [INSPIRE].

[60] C. Anastasiou et al., High precision determination of the gluon fusion Higgs boson cross-section at the LHC, JHEP 05 (2016) 058 [arXiv: 1602.00695] [INSPIRE].

[61] R.V. Harlander, T. Neumann, K.J. Ozeren and M. Wiesemann, Top-mass effects in differential Higgs production through gluon fusion at order $\alpha_{s}^{4}$, JHEP 08 (2012) 139 [arXiv:1206.0157] [INSPIRE].

[62] T. Neumann and M. Wiesemann, Finite top-mass effects in gluon-induced Higgs production with a jet-veto at NNLO, JHEP 11 (2014) 150 [arXiv:1408.6836] [INSPIRE].

[63] P. Artoisenet et al., A framework for Higgs characterisation, JHEP 11 (2013) 043 [arXiv: 1306.6464] [INSPIRE].

[64] F. Demartin, F. Maltoni, K. Mawatari, B. Page and M. Zaro, Higgs characterisation at $N L O$ in QCD: CP properties of the top-quark Yukawa interaction, Eur. Phys. J. C 74 (2014) 3065 [arXiv: 1407.5089] [inSPIRE].

[65] J. Alwall et al., The automated computation of tree-level and next-to-leading order differential cross sections and their matching to parton shower simulations, JHEP $\mathbf{0 7}$ (2014) 079 [arXiv: 1405.0301] [INSPIRE].

[66] K.G. Chetyrkin, B.A. Kniehl and M. Steinhauser, Virtual top quark effects on the $H \rightarrow b \bar{b}$ decay at next-to-leading order in QCD, Phys. Rev. Lett. 78 (1997) 594 [hep-ph/9610456] [INSPIRE].

[67] K.G. Chetyrkin, B.A. Kniehl and M. Steinhauser, Three loop $\mathcal{O}\left(\alpha_{s}^{2} G_{F} M_{t}^{2}\right)$ corrections to hadronic Higgs decays, Nucl. Phys. B 490 (1997) 19 [hep-ph/9701277] [INSPIRE].

[68] K.G. Chetyrkin, B.A. Kniehl and M. Steinhauser, Decoupling relations to $\mathcal{O}\left(\alpha_{s}^{3}\right)$ and their connection to low-energy theorems, Nucl. Phys. B 510 (1998) 61 [hep-ph/9708255] [INSPIRE].

[69] R. Frederix, S. Frixione, V. Hirschi, D. Pagani, H.S. Shao and M. Zaro, The automation of next-to-leading order electroweak calculations, JHEP 07 (2018) 185 [arXiv:1804.10017] [INSPIRE]. 
[70] C. Degrande, R. Frederix, V. Hirschi, M. Ubiali, M. Wiesemann and M. Zaro, Accurate predictions for charged Higgs production: Closing the $m_{H^{ \pm}} \sim m_{t}$ window, Phys. Lett. B 772 (2017) 87 [arXiv:1607.05291] [INSPIRE].

[71] S. Dittmaier, M. Krämer, A. Muck and T. Schluter, MSSM Higgs-boson production in bottom-quark fusion: Electroweak radiative corrections, JHEP 03 (2007) 114 [hep-ph/0611353] [INSPIRE].

[72] S. Dawson, C.B. Jackson and P. Jaiswal, SUSY QCD Corrections to Higgs-b Production: Is the $\Delta_{b}$ Approximation Accurate?, Phys. Rev. D 83 (2011) 115007 [arXiv:1104.1631] [INSPIRE].

[73] NNPDF collaboration, Parton distributions from high-precision collider data, Eur. Phys. J. C 77 (2017) 663 [arXiv:1706.00428] [INSPIRE].

[74] A. Buckley et al., LHAPDF6: parton density access in the LHC precision era, Eur. Phys. J. C 75 (2015) 132 [arXiv:1412.7420] [InSPIRE].

[75] R. Frederix, S. Frixione, V. Hirschi, F. Maltoni, R. Pittau and P. Torrielli, Four-lepton production at hadron colliders: aMC@NLO predictions with theoretical uncertainties, JHEP 02 (2012) 099 [arXiv: 1110.4738] [INSPIRE].

[76] P. Marquard, A.V. Smirnov, V.A. Smirnov and M. Steinhauser, Quark Mass Relations to Four-Loop Order in Perturbative QCD, Phys. Rev. Lett. 114 (2015) 142002 [arXiv:1502.01030] [INSPIRE].

[77] A.L. Kataev and V.S. Molokoedov, On the flavour dependence of the $\mathcal{O}\left(\alpha_{s}^{4}\right)$ correction to the relation between running and pole heavy quark masses, Eur. Phys. J. Plus 131 (2016) 271 [arXiv: 1511.06898] [INSPIRE].

[78] LHC Higgs Cross Section Working Group collaboration, Handbook of LHC Higgs Cross Sections: 4. Deciphering the Nature of the Higgs Sector, arXiv:1610.07922 [INSPIRE].

[79] M. Cacciari, G.P. Salam and G. Soyez, The anti- $k_{t}$ jet clustering algorithm, JHEP 04 (2008) 063 [arXiv: 0802.1189] [InSPIRE].

[80] M. Cacciari, G.P. Salam and G. Soyez, FastJet User Manual, Eur. Phys. J. C 72 (2012) 1896 [arXiv:1111.6097] [INSPIRE].

[81] F. Bishara, U. Haisch, P.F. Monni and E. Re, Constraining Light-Quark Yukawa Couplings from Higgs Distributions, Phys. Rev. Lett. 118 (2017) 121801 [arXiv:1606.09253] [INSPIRE].

[82] F. Cascioli, P. Maierhöfer, N. Moretti, S. Pozzorini and F. Siegert, NLO matching for t $\bar{t} b \bar{b}$ production with massive b-quarks, Phys. Lett. B 734 (2014) 210 [arXiv:1309.5912] [INSPIRE].

[83] T. Ježo, J.M. Lindert, N. Moretti and S. Pozzorini, New NLOPS predictions for $t \bar{t}+b-j e t$ production at the LHC, Eur. Phys. J. C 78 (2018) 502 [arXiv: 1802.00426] [INSPIRE].

[84] E. Bagnaschi, F. Maltoni, A. Vicini and M. Zaro, Lepton-pair production in association with $a b \bar{b}$ pair and the determination of the $W$ boson mass, JHEP 07 (2018) 101 [arXiv: 1803.04336] [INSPIRE].

[85] LHC Higgs Cross Section Working Group collaboration, LHC HXSWG interim recommendations to explore the coupling structure of a Higgs-like particle, arXiv:1209.0040 [INSPIRE]. 
[86] L. Buonocore, P. Nason and F. Tramontano, Heavy quark radiation in NLO+PS POWHEG generators, Eur. Phys. J. C 78 (2018) 151 [arXiv:1711.06281] [InSPIRE].

[87] T. Inami, T. Kubota and Y. Okada, Effective Gauge Theory and the Effect of Heavy Quarks in Higgs Boson Decays, Z. Phys. C 18 (1983) 69 [inSPIRE].

[88] S. Dawson, Radiative corrections to Higgs boson production, Nucl. Phys. B 359 (1991) 283 [INSPIRE].

[89] V. Hirschi, R. Frederix, S. Frixione, M.V. Garzelli, F. Maltoni and R. Pittau, Automation of one-loop QCD corrections, JHEP 05 (2011) 044 [arXiv:1103.0621] [INSPIRE].

[90] A.B. Goncharov, Multiple polylogarithms, cyclotomy and modular complexes, Math. Res. Lett. 5 (1998) 497 [arXiv:1105.2076] [InSPIRE].

[91] A.B. Goncharov, Multiple polylogarithms and mixed Tate motives, math/0103059.

[92] J. Kuipers, T. Ueda, J.A.M. Vermaseren and J. Vollinga, FORM version 4.0, Comput. Phys. Commun. 184 (2013) 1453 [arXiv:1203.6543] [InSPIRE].

[93] P. Nogueira, Automatic Feynman graph generation, J. Comput. Phys. 105 (1993) 279 [INSPIRE].

[94] R.N. Lee, LiteRed 1.4: a powerful tool for reduction of multiloop integrals, J. Phys. Conf. Ser. 523 (2014) 012059 [arXiv: 1310.1145] [INSPIRE].

[95] B. Jantzen, A.V. Smirnov and V.A. Smirnov, Expansion by regions: revealing potential and Glauber regions automatically, Eur. Phys. J. C 72 (2012) 2139 [arXiv:1206.0546] [INSPIRE].

[96] V.A. Smirnov, Applied asymptotic expansions in momenta and masses, Springer Tracts Mod. Phys. 177 (2002) 1 [inSPIRE].

[97] C. Anastasiou, C. Duhr, F. Dulat and B. Mistlberger, Soft triple-real radiation for Higgs production at N3LO, JHEP 07 (2013) 003 [arXiv:1302.4379] [INSPIRE].

[98] C. Duhr and F. Dulat, Polylogtools, private code (2014).

[99] C.W. Bauer, A. Frink and R. Kreckel, Introduction to the GiNaC framework for symbolic computation within the C++ programming language, J. Symb. Comput. 33 (2000) 1 [cs/0004015] [INSPIRE].

[100] A.V. Smirnov, FIESTA4: Optimized Feynman integral calculations with GPU support, Comput. Phys. Commun. 204 (2016) 189 [arXiv:1511.03614] [INSPIRE]. 